\title{
Hierarchically refined isogeometric analysis of trimmed shells
}

\author{
Luca Coradello ${ }^{*, \dagger, 1}$, Davide D’Angella $a^{\ddagger \dagger, 2,3}$, Massimo Carraturo ${ }^{2,4}$, Josef \\ $\mathrm{Kiendl}^{5}$, Stefan Kollmannsberger ${ }^{2}$, Ernst Rank $^{2,3}$, and Alessandro Reali ${ }^{3,4}$ \\ ${ }^{1}$ Chair of Numerical Modelling and Simulation, École Polytechnique Fédérale de Lausanne, Switzerland \\ ${ }^{2}$ Chair of Computational Modeling and Simulation, Technische Universität München, Germany \\ ${ }^{3}$ Institute for Advanced Study, Technische Universität München, Germany \\ ${ }^{4}$ Dipartimento di Ingegneria Civile e Architettura, Università degli Studi di Pavia, Italy \\ ${ }^{5}$ Department of Marine Technology, Norwegian University of Science and Technology, Norway
}

\begin{abstract}
This work focuses on the study of several computational challenges arising when trimmed surfaces are directly employed for the isogeometric analysis of Kirchhoff-Love shells. To cope with these issues and to resolve mechanical and/or geometrical features of interest, we exploit the local refinement capabilities of hierarchical B-Splines. In particular, we show numerically that local refinement is suited to effectively impose Dirichlet-type boundary conditions in a weak sense, where this easily allows to overcome the issue of over-constraining of trimmed elements. Moreover, we highlight how refinement can alleviate the spurious coupling stemming from disjoint supports of basis functions in the presence of "small" trimmed geometrical features such as thin holes. These phenomena are particularly pronounced in surface models defined by complex trimming patterns and with details at different scales, where we show through several numerical examples the benefits and computational efficiency of the proposed methodology.
\end{abstract}

\section{Introduction}

The standard procedure in computational engineering is, starting from a geometric Computer Aided Design (CAD) model, to produce a proper mesh suitable to perform a finite element analysis. It is well known that for complex geometries, the meshing process is cumbersome and highly time-consuming [14. Furthermore, the created mesh is in most cases only an approximation of the real design, which can deteriorate the accuracy of the solution. To overcome these issues, IsoGeometric Analysis (IGA) was introduced in the first pioneering work [32] in 2005. The pivotal idea in IGA is to exploit the same mathematical entities used in CAD for the geometry description, for instance B-Splines and NonUniform Rational B-Splines (NURBS), as basis functions for the analysis. Additionally, employing smooth functions like B-splines or variations thereof as a basis for the solution field has shown excellent properties in many mathematical and engineering applications. For a detailed review of the method and its applications and mathematical foundations, the reader is referred to [1, 14, 15, 32]. Moreover, it has been shown that for many applications the higher global continuity achievable with B-Splines yields a better accuracy per degree-of-freedom and, more importantly in the scope of this

\footnotetext{
*luca.coradello@epfl.ch

${ }^{\dagger}$ Both authors contributed equally to this work

${ }^{\ddagger}$ davide.dangella@tum.de
} 
work, it allows to discretize higher-order variational problems in their primal form, e.g. KirchhoffLove plates and shells or Cahn-Hilliard problems [25, 34, 36, 40, 47].

However, there are still several open questions that need to be addressed for IGA to become competitive for industrial applications. Indeed, the gap between geometric design and numerical analysis is yet to be fully closed and a general methodology capable of handling trimming, dirty geometries and volumetric mesh generation currently represents a thriving area of research, see for instance [3, 41, 44, 50, 56].

Nevertheless, a significant amount of work has been performed for the analysis of trimmed surfaces in the context of dimensionally-reduced formulations such as plates and shells, where the analysis makes use directly of the surface representation, hence greatly simplifying the interoperability with CAD. The reader is referred to the pioneering works [8, 52, 53] and [10, 28] for more insights.

An additional challenge comes from the tensor product nature of B-splines which poses severe limitations to local refinement. Indeed, it is common in practice to require higher discretization accuracy in selected areas where small geometric features or localized mechanical responses play an important role in the analysis. In standard h-refinement of B-splines, the refinement propagates throughout the entire patch, yielding a loss of locality. Several methods have been proposed to overcome this drawback, such as hierarchical B-Splines (HB) [21, 26, 37] and their recent variant denoted by truncated hierarchical B-Splines (THB) [23, 24, T-Splines [6], LR-Splines [18]. Also the inverse operation of refinement (known as coarsening), which plays an important role for computational efficiency, proves to be a non-trivial task and sparked research, see for instance [9, 22, 43].

In this work we demonstrate the importance of local refinement in the isogeometric analysis of trimmed thin-walled structures where the refinement capabilities are achieved thanks to hierarchical B-Splines. The latter is an important feature, since it allows to efficiently capture localized traits of the solution. Furthermore, we show that local refinement improves the accuracy of the imposition of (weak) penalty boundary conditions on complex trimmed boundaries and mitigates the effect of overconstraining the solution space. Moreover, we tackle the issue of the spurious coupling introduced in the solution field arising when "small" (compared to the element size) features are present in the geometry (e.g. thin holes), where again the use of local refinement proves to be an effective remedy.

The focus is also put on how the proposed combination of methods brings one step closer the integration between boundary representation (B-Rep)-based design and shell analysis, namely tackling the open question of how to properly treat trimmed surfaces. Instead of producing conforming T-Spline meshes [10, 42, we shift the effort to accurately integrate trimmed elements. This topic has already been analyzed in [8, 28, 50], and we use a similar methodology for the creation of boundaryconforming integration cells. In particular, we employ a technique similar to what is proposed in 28. Indeed, for this task, we extend the Finite Cell Method (FCM) to the analysis of thin structures, developing the ideas first introduced in [46] and applying the boundary-conforming adaptive integration scheme described in [38, 39] to trimmed surfaces, where the main difference with respect to 28] lies in the decomposition algorithm for complex trimming scenarios. We characterize the Finite Cell Method as an immersed method, which makes use of high-order shape functions. FCM has been successfully applied in combination with high-order polynomials, such as Lagrange or integrated Legendre, to several practical problems of linear and non-linear elasticity, thermo-elasticity [57, transient elastodynamics [20], biomechanical applications [48, elasto-plasticity [2], contact mechanics [7]. Moreover, it has been shown that this method works well also in combination with B-Splines and variants thereof [50]. For a detailed review of the method, the reader is referred to [19, 45, 51] and references therein.

Then, based on the work [16], we show how local refinement can be easily implemented into the proposed workflow by combining hierarchical B-Splines together with FCM. Moreover, we highlight how the use of refinement alleviates several issues stemming from trimming. Finally, starting from 
geometrical information exported from the CAD software Rhinoceros ${ }^{1}$ in STEP format [33], we show through several numerical examples the robustness and wide range of applicability of the presented methodology, highlighting its generality and flexibility.

This work is structured as follows. Section 2 summarizes the methods which constitute the framework, namely hierarchical B-Splines to allow for local refinement, the Kirchhoff-Love shell formulation for the analysis of thin structures, and the Finite Cell Method for handling trimming in an immersed sense. Then, in Section 3 we highlight by means of several examples the advantages of using local refinement to resolve localized features of the solution and of the geometry, and to weakly impose boundary conditions of Dirichlet-type. In Section 4 we present various numerical results on complex designs. Finally, in Section 5 we draw some conclusions.

\section{Methods}

In the following section, all methods that have been combined in the presented framework are briefly summarized. As previously described, all these methods are already well established in the literature; nevertheless, they are brought together for the first time within the same numerical framework in the present work. This leads to a general and powerful tool for the isogeometric analysis of trimmed surfaces.

\subsection{Hierarchical B-Splines}

In this subsection, the concept of hierarchical B-Spline basis $\mathcal{H B}$, firstly introduced in [37, 55], is presented following the notation in [16. This allows to build a basis that is locally refined and therefore to overcome the limitations intrinsic to the tensor-product nature of B-Splines and NURBS.

Let $V^{0} \subset V^{1} \subset \cdots \subset V^{N}$ be a sequence of nested spaces of splines defined on a rectangular parametric domain $\widehat{\Omega}$. Each space $V^{l}, l=0, \ldots, N$ is spanned by the B-spline basis $\mathcal{B}^{l}$ of degree $p$ defined on the grid $\mathcal{Q}^{l}$, associated to level $l$. Similarly, we can define a hierarchy of subdomains up to depth $N$ as $\Omega^{N}:=\left\{\Omega^{0}, \Omega^{1}, \ldots, \Omega^{N}\right\}$ if the following holds:

$$
\widehat{\Omega}=\Omega^{0} \supset \Omega^{1} \supset \ldots \supset \Omega^{N-1} \supset \Omega^{N}=\emptyset .
$$

Let us now define the hierarchical B-Spline basis $\mathcal{H B}$. Given a sequence of nested spaces $\left\{V^{l}\right\}_{l=0, \ldots, N}$, their corresponding B-Spline bases $\left\{\mathcal{B}^{l}\right\}_{l=0, \ldots, N}$ and a hierarchy of subdomains $\boldsymbol{\Omega}^{N}$, we characterize $\mathcal{H B}$ in a recursive fashion as:

$$
\begin{aligned}
\mathcal{H} \mathcal{B}^{0}= & \mathcal{B}^{0} \\
\mathcal{H} \mathcal{B}^{l+1}= & \left\{b \in \mathcal{H B}^{l} \mid \operatorname{supp} b \not \subset \Omega^{l+1}\right\} \cup \\
& \left\{b \in \mathcal{B}^{l+1} \mid \operatorname{supp} b \subset \Omega^{l+1}\right\}, \quad l=0, \ldots, N-2 .
\end{aligned}
$$

It was proven in [55] that this set of B-Splines is composed of linearly independent functions. At this point, let us define the discrete space $X_{h}$ as follows:

$$
X_{h}=\operatorname{span}\left\{b \circ \mathbf{S}^{-1} \mid b \in \mathcal{H B}\right\},
$$

where $\boldsymbol{S}$ denotes the B-Spline parametrization of the surface.

Remark 1 The construction of $\mathcal{H B}$ can be generalized to trimmed geometries (see [4, 31]), and it was proven in [30, Section 4.5] that they retain their linear independence and therefore they form a

\footnotetext{
${ }^{1}$ www.rhino3d.com
} 
suitable basis for the analysis on trimmed domains.

\subsection{Kirchhoff-Love shells}

In this work, we make use of the rotation-free Kirchhoff-Love shell element. In the following subsection, the formulation is briefly introduced, following closely the derivation in [12, 34]. We remark that to obtain a rotation-free formulation for the Kirchhoff-Love problem at least global $C^{1}$-continuity of the basis is required, which is readily achievable using, e.g., (hierarchical) B-Splines of maximum continuity and degree $p>1$.

\subsubsection{The weak form of the problem}

Applying standard variational calculus, the weak form of the Kirchhoff-Love problem reads [12, 34]:

$$
\begin{aligned}
& \text { Find } \boldsymbol{u} \in V \text { such that } \\
& a(\boldsymbol{u}, \boldsymbol{v})=F(\boldsymbol{v}) \quad \forall \boldsymbol{v} \in V,
\end{aligned}
$$

where, for ease of notation and without loss of generality, we assume the fully clamped case and therefore $V=H_{0}^{2}(\Omega)$. We remark that when different boundary conditions are prescribed a corresponding different space $V$ should be taken into account [11]. Then, the linear and bilinear forms read:

$$
\begin{aligned}
a(\boldsymbol{u}, \boldsymbol{v}) & =\int_{\Omega} \boldsymbol{\varepsilon}(\boldsymbol{v}): \boldsymbol{n}(\boldsymbol{u}) d \Omega+\int_{\Omega} \boldsymbol{\kappa}(\boldsymbol{v}): \boldsymbol{m}(\boldsymbol{u}) d \Omega \\
F(\boldsymbol{v}) & =\int_{\Omega} \boldsymbol{v} \cdot \boldsymbol{b} d \Omega
\end{aligned}
$$

where $\varepsilon, \boldsymbol{\kappa}$ denote the membrane and bending strain tensors, respectively, and $\boldsymbol{n}, \boldsymbol{m}$ are their energetically conjugate stress resultants, and $\boldsymbol{b}$ represents the external body load. For a detailed review of the Kirchhoff-Love formulation, we refer to [34].

Using the classical Bubnov-Galerkin approach, we can write the following discrete weak formulation of (1):

$$
\begin{aligned}
& \text { Find } \boldsymbol{u}_{h} \in V_{h} \subset V \text { such that } \\
& a\left(\boldsymbol{u}_{h}, \boldsymbol{v}_{h}\right)=F\left(\boldsymbol{v}_{h}\right) \quad \forall \boldsymbol{v}_{h} \in V_{h} .
\end{aligned}
$$

We remind the reader that in general the choice of discrete space $V_{h} \subset X_{h}$ depends on the boundary conditions of the problem at hand. Moreover, we recall that due to the requirements on the discrete admissible space for the displacement field, the basis functions must be at least $C^{1}$ continuous globally such that the bending operators $\boldsymbol{\kappa}$ and $\boldsymbol{m}$ are well-defined.

\subsection{Trimming}

In the next subsection, it is shown how the numerical challenges stemming from trimmed geometries can be directly tackled by using an immersed method, such as the Finite Cell Method (FCM). In particular, an adaptive integration technique that resolves trimming at the integration level is presented, based on the work of [46] and in the spirit of what proposed in [8, 28] in the framework of isogeometric trimmed shells.

Let us characterize trimming as a basic boolean operation, which allows for an easy description of complex geometries and constitutes a standard in most commercial CAD softwares. Albeit its simple conceptual definition, trimming poses severe limitations to the interoperability between CAD and the analysis, where we refer to the review article [44]. Indeed, when we perform a trimming operation in a 
CAD software, the visualization of the resulting surface is modified but its underlying mathematical description remains unchanged. This means that if we utilize directly the basis functions defined by the geometry, they are not conforming to the physical boundary. This concept resembles the methodology employed in the so-called immersed or embedded domain methods.

\subsubsection{The FCM formulation of the Kirchhoff-Love problem}

Employing the FCM fundamental idea of an embedded domain approach, let us extend the physical domain $\Omega^{\text {phy }}$ by a fictitious part, denoted by $\Omega^{\text {fict }}$, such that $\Omega=\Omega^{\text {phy }} \cup \Omega^{\text {fict }}$ with $\Omega^{\text {phy }} \cap \Omega^{\text {fict }}=\emptyset$. Note that since $\Omega$ can be chosen arbitrarily, simple shapes are obviously advantageous since they can be trivially discretized with a Cartesian grid. Furthermore, note that with the introduction of a fictitious domain, we no longer have a mesh conforming to the physical boundary. Indeed, as typical of immersed methods, Dirichlet-type boundary conditions are usually enforced in a weak sense. In this work, we consider weak constraints applied by means of the penalty method [5]. In particular, a weak displacement and rotational boundary condition terms are added and the weak form of the discrete Kirchhoff-Love problem (2) can be reformulated as:

Find $\boldsymbol{u}_{h} \in V_{h}$ such that

$$
a_{\alpha}\left(\boldsymbol{u}_{h}, \boldsymbol{v}_{h}\right)+b^{\operatorname{disp}, k}\left(u_{h}^{k}, v_{h}^{k}\right)+b^{\text {rot }}\left(\boldsymbol{u}_{h}, \boldsymbol{v}_{h}\right)=F_{\alpha}\left(\boldsymbol{v}_{h}\right) \quad \forall \boldsymbol{v}_{h} \in V_{h},
$$

where:

$$
\begin{aligned}
a_{\alpha}\left(\boldsymbol{u}_{h}, \boldsymbol{v}_{h}\right) & =\int_{\Omega} \alpha \boldsymbol{\varepsilon}\left(\boldsymbol{v}_{h}\right): \boldsymbol{n}\left(\boldsymbol{u}_{h}\right) d \Omega+\int_{\Omega} \alpha \boldsymbol{\kappa}\left(\boldsymbol{v}_{h}\right): \boldsymbol{m}\left(\boldsymbol{u}_{h}\right) d \Omega \\
b^{\mathrm{disp}, k}\left(u_{h}^{k}, v_{h}^{k}\right) & =\int_{\Gamma_{g^{k}}} \beta^{\mathrm{disp}}\left(u_{h}^{k}-g^{k}\right) v_{h}^{k} d \Gamma \\
b^{\mathrm{rot}}\left(\boldsymbol{u}_{h}, \boldsymbol{v}_{h}\right) & =\int_{\Gamma_{\theta}} \beta^{\text {rot }} \boldsymbol{d} \cdot \boldsymbol{\Phi}\left(\boldsymbol{v}_{h}\right) \boldsymbol{\Phi}\left(\boldsymbol{u}_{h}\right) \cdot \boldsymbol{d} d \Gamma \\
F_{\alpha}\left(\boldsymbol{v}_{h}\right) & =\int_{\Omega} \alpha \boldsymbol{v}_{h} \cdot \boldsymbol{b} d \Omega \\
\alpha(\boldsymbol{x}) & = \begin{cases}1 & \text { if } \boldsymbol{x} \in \Omega^{\mathrm{phy}} \\
0 & \text { if } \boldsymbol{x} \in \Omega^{\text {fict }},\end{cases}
\end{aligned}
$$

where $\alpha(\boldsymbol{x})$ acts as a domain indicator and $k$ sums over the displacement components. Note that in practice, the value of $\alpha$ inside $\Omega^{\text {fict }}$ is chosen to be numerically small, such that extremely illconditioned element stiffness matrices are avoided for those cells that are partly lying in $\Omega^{\text {fict }}$, denoted in the following as cut cells. Then, $\boldsymbol{\Phi}(\cdot)$ denotes the angle between the normal to the surface in its reference and deformed configuration, namely $\boldsymbol{\Phi}(\cdot)=\boldsymbol{a}_{\mathbf{3}}-\boldsymbol{A}_{\mathbf{3}}$, and $\boldsymbol{d}$ represents the normal (lying in the plane tangent to the surface) to the constrained boundary $\Gamma_{\theta}$. For a detailed review, we refer to [27, 29]. Moreover, $g^{k}$ denotes the prescribed displacement on $\Gamma_{g^{k}}$ and $\beta^{\text {disp }}, \beta^{\text {rot }} \in \mathbb{R}$ are the displacement and rotational penalty parameters, respectively. Typically, they are scaled with the material parameters and element size [54], where following [29] we use

$$
\begin{aligned}
\beta^{\text {disp }} & =\bar{\beta} \frac{E t}{h\left(1-\nu^{2}\right)} \\
\beta^{\text {rot }} & =\bar{\beta} \frac{E t^{3}}{12 h\left(1-\nu^{2}\right)},
\end{aligned}
$$

where $E, \nu, t$, and $h$ are the Young modulus, the Poisson ratio, the thickness, and the element size, respectively, while $\bar{\beta}$ is a user-defined parameter. For non-uniform refinement, $h$ is heuristically 
chosen as the smallest element size.

Remark 2 It is worth noting that for surfaces we do not directly apply the FCM methodology to the space $\Omega \subset \mathbb{R}^{3}$. Instead for simplicity and robustness, following [46] and similar to [8, [28], the trimming curves are either directly exported in the parametric space of the surface or are pulled back to the parametric image of $\Omega$, previously denoted by $\hat{\Omega} \subset \mathbb{R}^{2}$. Then, the same FCM rationale is applied in the parameter space of the shell. Hence, the treatment of trimmed surfaces in the physical space is tackled directly in the parameter space at the integration level.

Remark 3 We highlight that we do not restrict our analysis to the case where boundary conditions are applied only weakly. Indeed, with a slight abuse of notation, we denote with $V_{h} \subset X_{h}$ the finite dimensional space that satisfies the strong Dirichlet boundary conditions of the problem, applied to the conforming part of the boundary $\partial \Omega \cap \partial \Omega^{\text {phy }}$.

\subsubsection{Smart tree integration for trimmed elements}

The domain indicator function $\alpha$ introduces a discontinuity in the integrals of the weak form (3) for those cells that are trimmed. As a consequence, the challenges arising from the generation of conforming meshes are shifted towards the accurate and efficient computation of these integrals. A method that has been successfully used is the so-called smart tree scheme, firstly introduced in [38]. This method falls into the category of boundary-conforming integration methods. Indeed, exploiting geometric information from the B-Rep model, the algorithm is able to decompose cut elements into boundary conforming integration cells, therefore yielding a highly accurate integration with a significant reduction of the number of Gauss points, for instance compared to space tree schemes. The information needed by the algorithm can be extracted directly from CAD softwares or can be obtained numerically by performing the pull-back of the physical trimming curves. For a detailed review of the method for two- and three-dimensional applications the reader is referred to [38, 39].

We utilize this algorithm for the creation of boundary-conforming integration meshes for those elements that are trimmed. The method is able to treat complex surfaces where sharp features and kinks are present and, in the scope pf this work, we extend it to handle directly B-Rep models from Rhinoceros.

In Algorithm 1, we summarize the smart tree algorithm (on a single cut cell) specialized to trimmed shells. In the following we assume that the trimming curves given as an input are defined in the parameter space of the surface.

If the decomposition can be performed, Algorithm 1 always yields a set of quadrilaterals and triangles, otherwise for too complex trimming patterns the fallback strategies exploits the space treebased integration. In particular, after each space tree step, the intersections between the integration cells and the trimming curves are recursively simplified up to the point where either the decomposition can be performed or the routine stops at a user-defined maximum depth of the space tree. Therefore, the robustness of the algorithm is enhanced and an accurate integration is guaranteed for arbitrary geometries. 


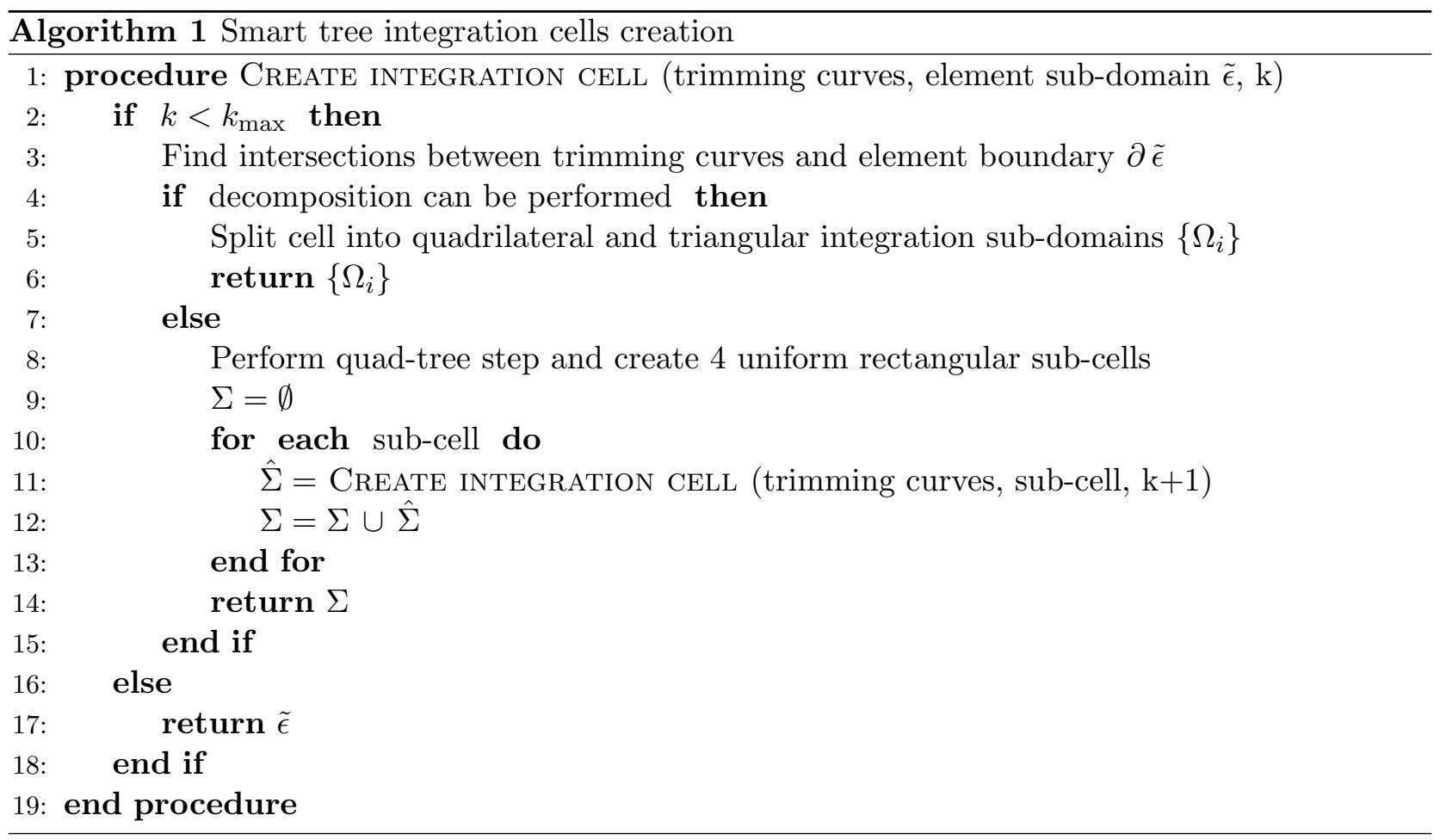

\section{Motivation to local refinement}

In this section, we motivate the use of local refinement for the analysis of trimmed geometries with the following arguments:

- removal of unphysical coupling between the sides of a thin hole.

- increased accuracy close to trimming boundaries subject to weak constraints;

- efficient approximation of localized deformations;

\subsection{Thin holes}

In case trimming curves define holes that are "thin" compared to the geometry knot spans, it can happen that the support of a basis function intersected with the physical domain $\Omega^{\text {phy }}$ is disconnected and composed of several disjoint sub-domains. As an example, the support of a function with two disjoint physical sub-domains is depicted in red in Figure 1b. This creates an unphysical coupling between the two sides of the hole, that generally results in an inaccurate mechanical response. These spurious effects become particularly severe when the local behavior of the structure is strongly determined by the geometry of the hole, as it is often the case for complex models. For instance, consider the setup illustrated in Figure 1a, where the geometry is modeled via a trimmed NURBS surface of degree $p=2$, which is exported from Rhinoceros together with a set of 32 trimming curves. It is worth remarking the complexity of the geometry at hand, which presents several thin sharp features and kinks in its description. For the analysis, homogeneous Dirichlet boundary conditions on the displacement are applied to the outer boundary of the violin via the penalty method, as described above, and a line load $\boldsymbol{F}=(0,0,-100)^{\top}$ is applied to the reentrant tip of the f-hole, such that the tip is pulled upwards. A direct analysis on the coarse geometry yields an unphysical response, where the maximum displacement is attained towards the center of the geometry (see Figure $1 \mathrm{c}$ obtained by setting $\bar{\beta}=10$ ), instead of the reentrant tip, as expected from engineering intuition.

In order to mitigate this issue, local refinement can be used to substitute functions with large support by functions with smaller support. In particular, the removal of the unphysical coupling 
is obtained when no support is composed of disjoint physical parts. To this end, let us consider the refinement procedure presented in Algorithm 2. We introduce a marking parameter $\gamma$, which represents the extension of the refinement area in the proximity of a given trimming curve. Note

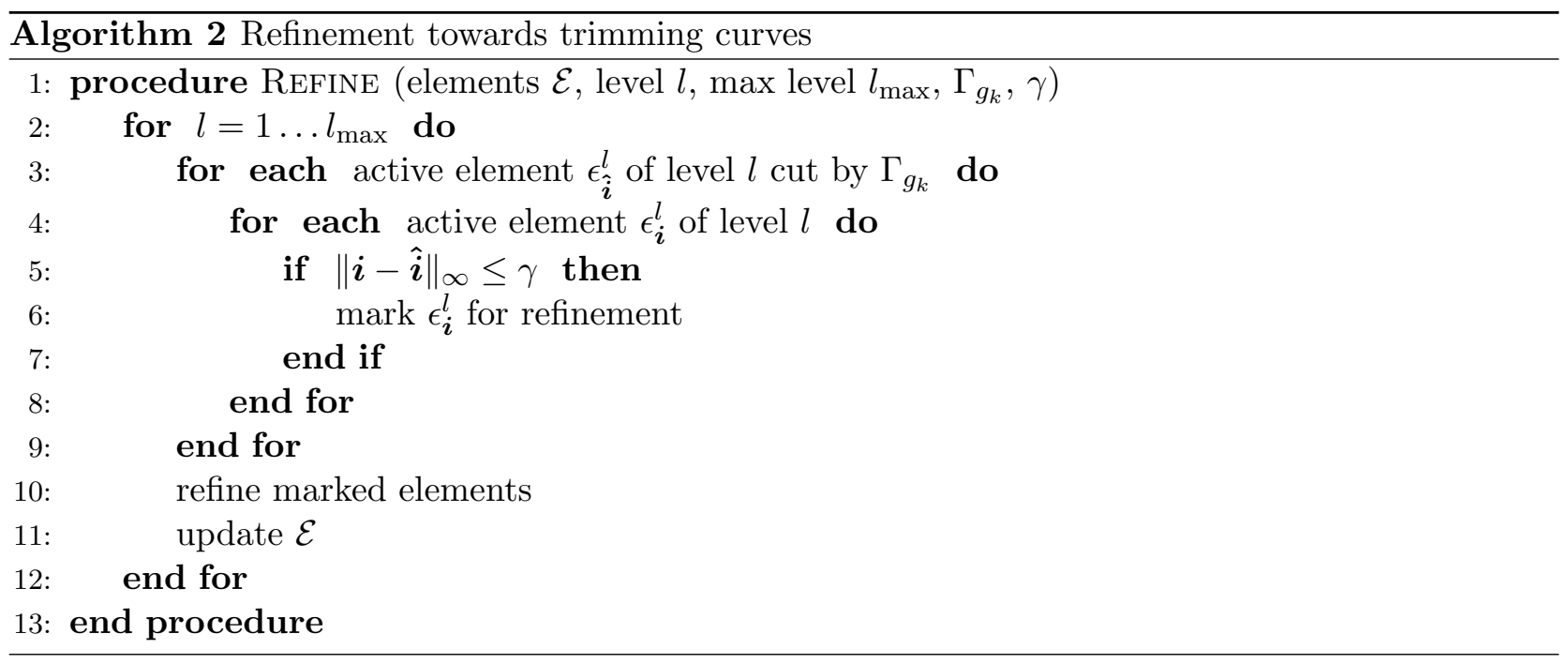

that for $\gamma=p$ or higher, only functions of the finest level have support on the trimming curve. By direct comparison between the size of the finest knot spans and the size of the hole, one can obtain the value for $l_{\max }$ that removes the unphysical coupling. For the current example we obtain a decoupling value $l_{\max }=5$ and a qualitatively correct response is observed in Figure $1 \mathrm{~d}$ for $\gamma=p=2$, where the maximum deflection occurs at the reentrant tip. In Figure 2a, the displacement at point A (marked with a blue dot in Figure 1b) is shown, where the latter point of interest is located at one of the geometrical kinks of the trimmed boundary. Note the sudden improvement in accuracy of the solution obtained for $l_{\max }=5$, while smaller choices of the maximum refinement level yield inaccurate results, since the spurious coupling is still present in the corresponding $\mathcal{H B}$ bases. Then, in Figure 2a, we compare the convergence behavior of the solution obtained by local refinement to the tensor product refinement constructed by Algorithm 2 with $\gamma=0$ and to a standard uniform tensorproduct refinement, obtained by recursively bisecting every knot span. The meshes corresponding to local and tensor-product refinements for $l_{\max }=5$ are depicted in Figures 3a and 3b, respectively. In Figure 2b we observe that all strategies converge towards a reference value for the displacement obtained from an overkill solution, where local refinement achieves a level of accuracy comparable to uniform refinement with substantially less degrees-of-freedom (DOFs).

Remark 4 To cope with the spurious coupling described above, we show the potential of the error estimator introduced in [13] to solve the issue by means of a simple example. We employ the geometry and problem setup depicted in Figure 4a, where a thin, elongated hole is trimmed away from a square plate. The displacement is set to zero on the external boundary of the geometry and the plate is subjected to a vertical point load $\boldsymbol{F}=(0,0,-1)^{\top}$ at the center of the structure. A reference displacement $\tilde{u}_{z}$ at point $B$ (marked with a blue dot in Figure $4 a$ ) has been obtained from an overkill solution on a uniformly-refined mesh with 199509 DOFs. The convergence plot presented in Figure $4 \mathrm{~b}$ confirms the importance of eliminating the unphysical coupling between the sides of thin holes. Moreover, the use of an error-driven strategy turns out to be an effective remedy, where the efficiency of the method is shown in Figures $4 \mathrm{C}$ and $4 \mathrm{~d}$, where the solution obtained on the initial coarse mesh and after $k=14$ iterations of the adaptive algorithm are graphically compared. Although these preliminary results are promising, further research is needed and currently under development. 


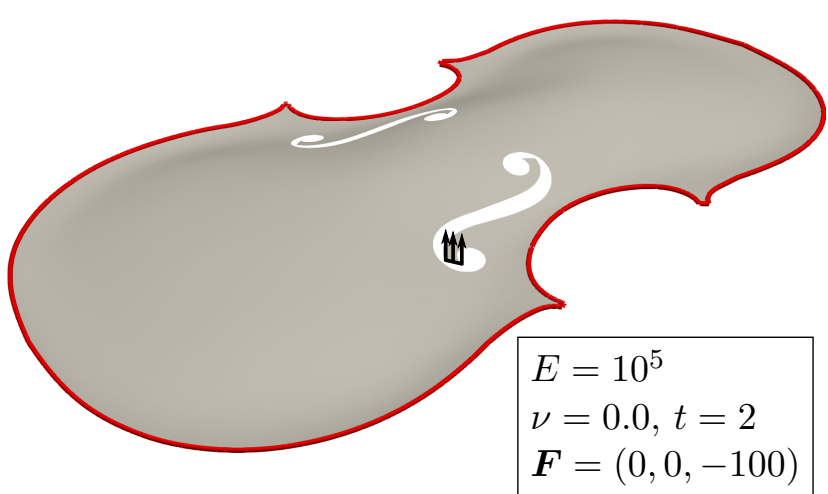

(a) Applied boundary conditions: homogeneous Dirichlet (red wire) and distributed load (arrows).

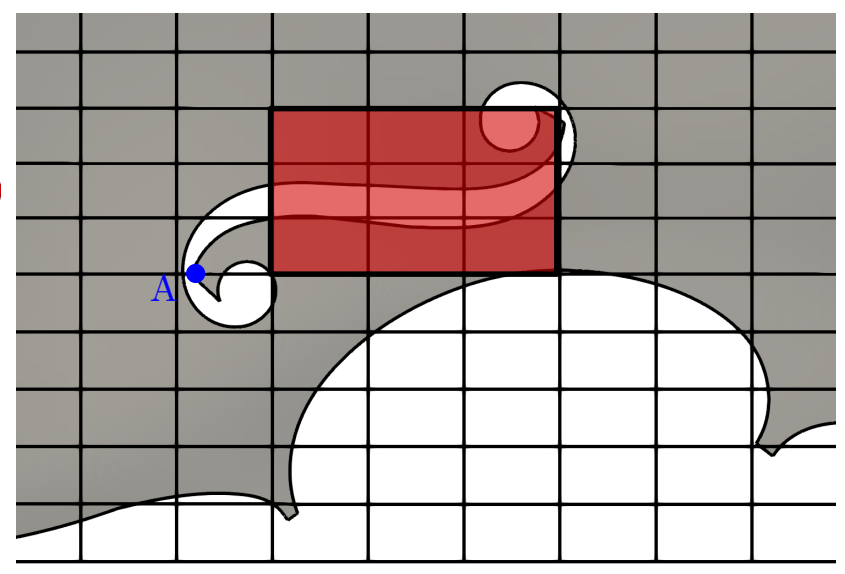

(b) Example of basis function support covering both sides of a thin hole ( $p=2$ and maximum continuity).

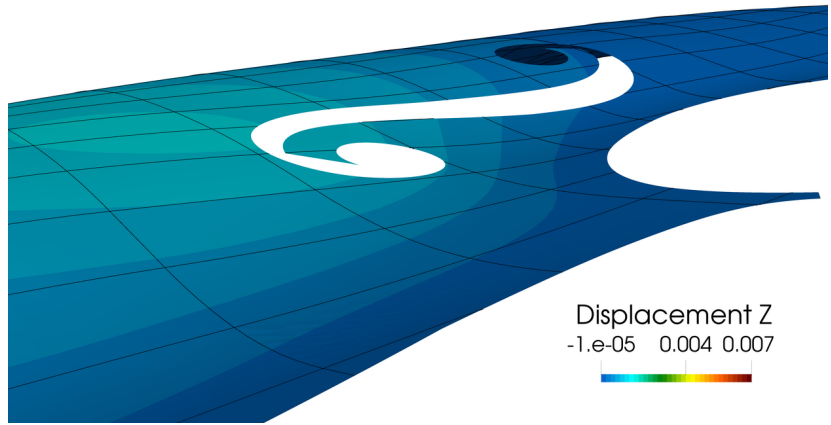

(c) Spurious and unphysical solution obtained from a direct analysis on the CAD model.

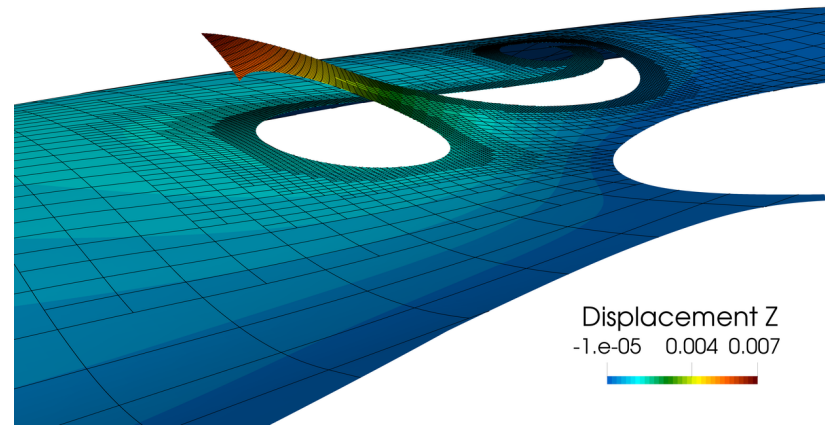

(d) Local refinement around the trimming curve removes the unphysical coupling.

Figure 1: Example problem on a complex trimmed geometry that shows the unphysical coupling occurring at the sides of thin holes.

\subsection{Weak constraints}

When imposing boundary conditions weakly on arbitrarily-shaped trimming curve, it can happen that the finite element space is not capable of both accurately satisfy the weak boundary conditions and approximate the numerical solution in the proximity of the trimming boundary. For complicated geometry and/or high penalty parameters $\beta^{\text {disp }}$ and $\beta^{\text {rot }}$ (without excessively deteriorating the conditioning of the system matrix), some elements will be over-constrained.

To illustrate this effect we consider the setup depicted in Figure 5a, where a uniformly distributed vertical load $\boldsymbol{F}=(0,0,-100)^{\top}$ is applied on the top surface, while zero displacement is enforced with a penalty term $\left(\bar{\beta}=10^{6}\right)$ on the internal curves, which feature kinks and areas of high curvature. In Figures $5 \mathrm{~b}$ and $5 \mathrm{c}$ it can be observed that the displacement and stress are artificially low on the elements cut by the trimming curve, and they spuriously follow the element boundaries. We remark that this effect is more pronounced in the proximity of complex trimming patterns.

Given a fixed mesh, it is in general desirable to balance the error in the application of boundary conditions and in the solution approximation. We refer to [17] for a discussion in the context of the Nietsche's method. However, in case it is not practical, or it is not known a priori how to optimally choose $\beta$, mesh refinement can be employed to potentially improve accuracy in both interior and boundary terms by enlarging the finite element space and intersecting the curve with smaller 


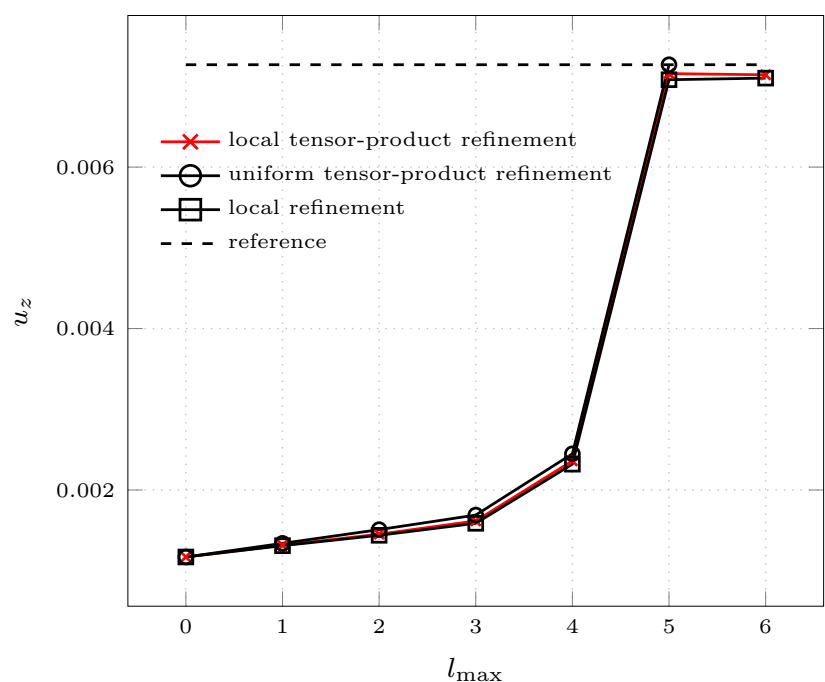

(a) Convergence plot of the numerical solution at point A, note the sudden change for $l_{\max }=5$.

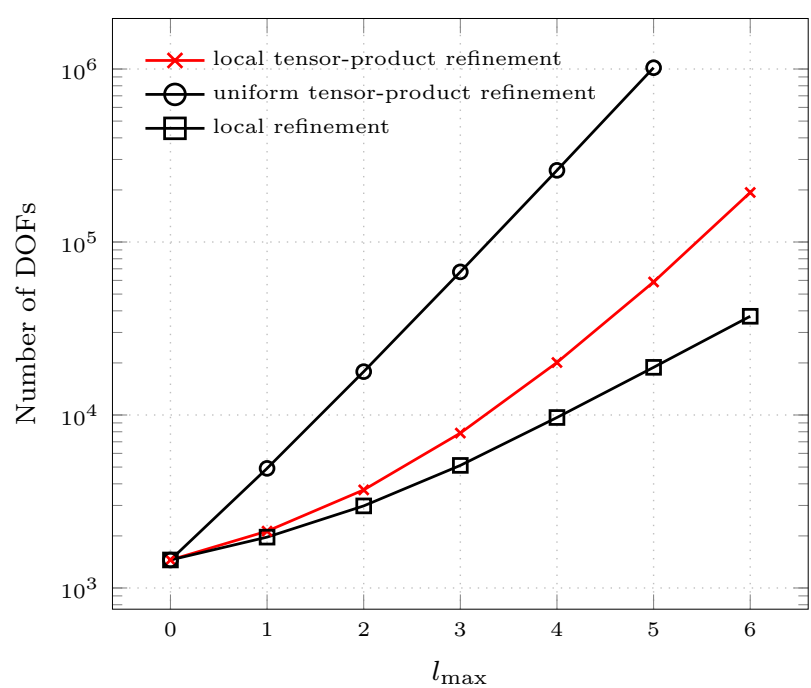

(b) Comparison of the number of DOFs as a function of the refinement parameter $l_{\max }$.

Figure 2: Convergence graphs for the violin example subjected to a line load.

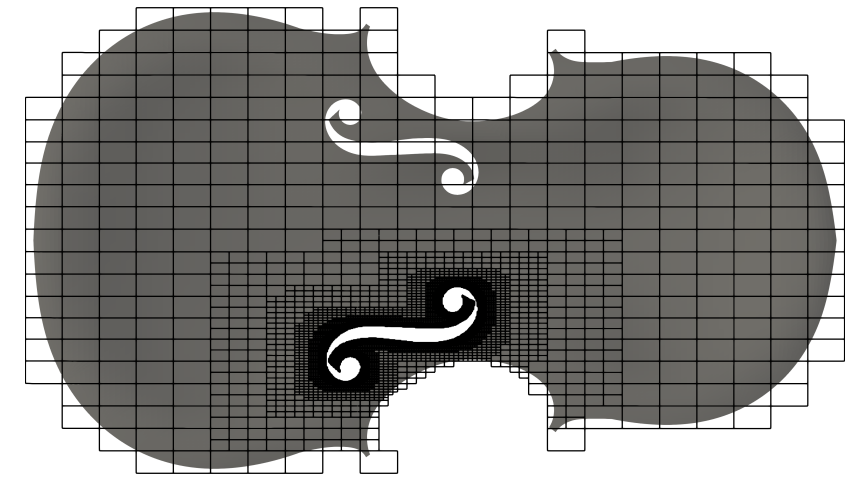

(a) Locally refined mesh.

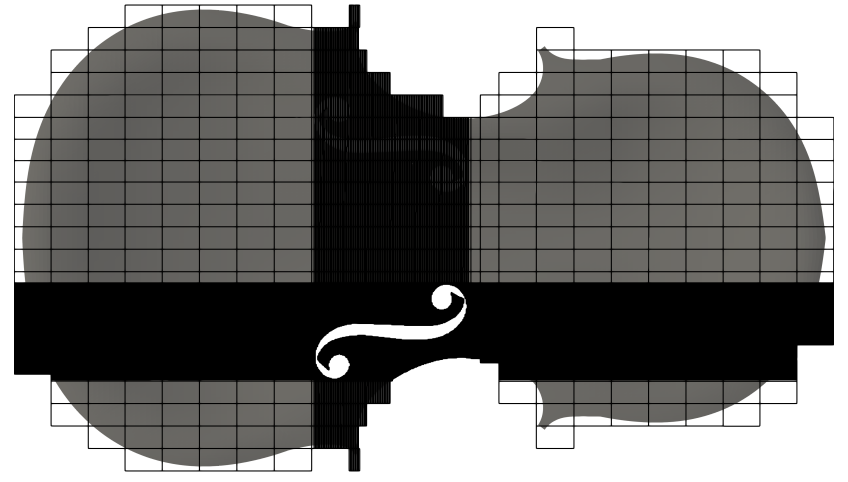

(b) Local tensor-product mesh.

Figure 3: Meshes obtained by local and local tensor-product refinements by setting $l_{\max }=5$. 


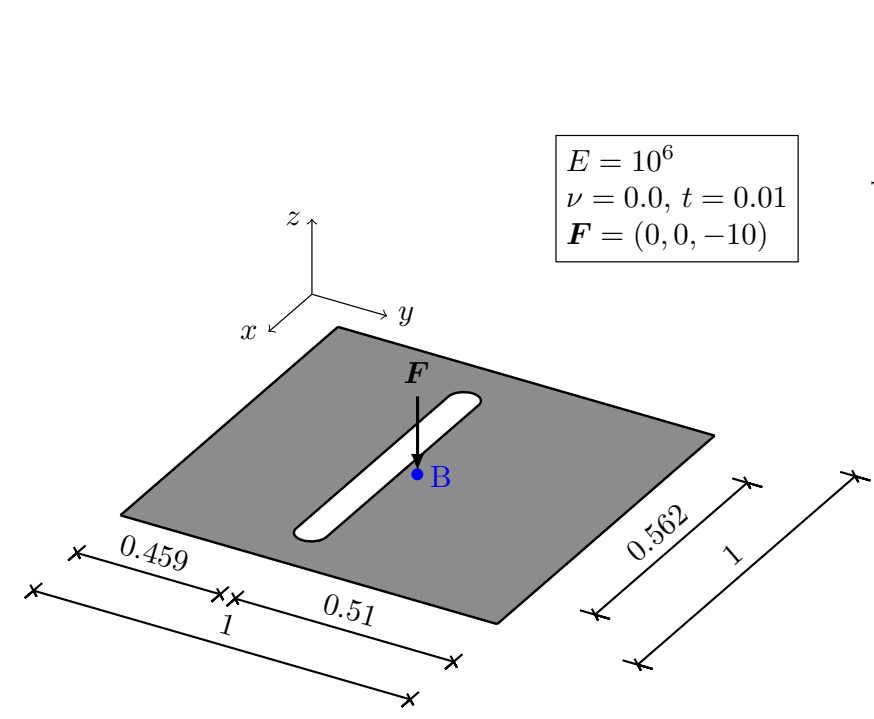

(a) Problem setup.

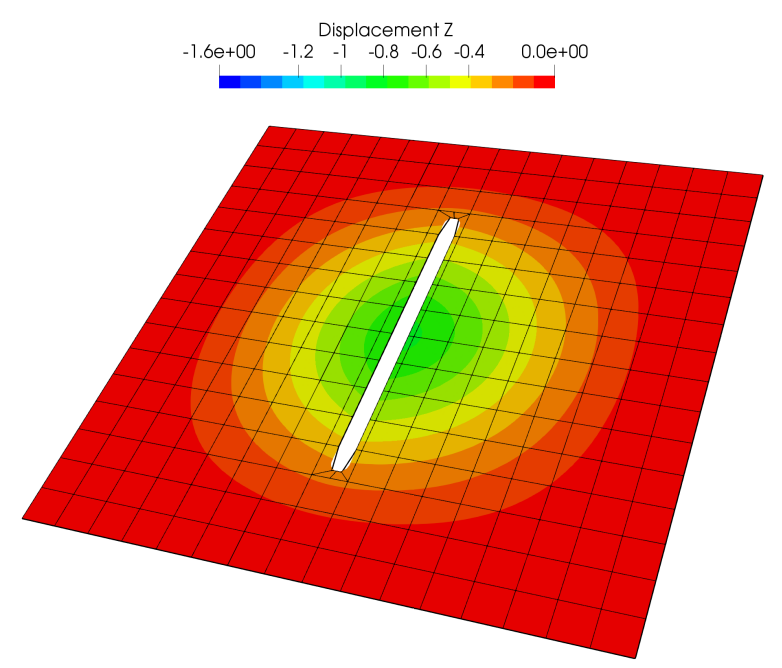

(c) Solution $u_{z}$ obtained on the coarse mesh, hierarchi- (d) cal B-Splines of degree $p=3$.

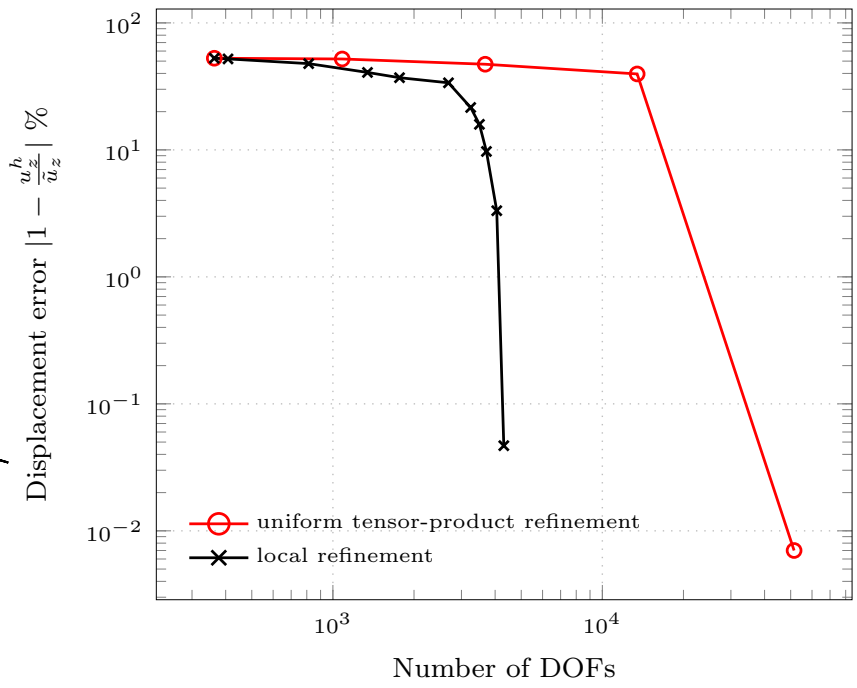

(b) Convergence plot of the normalized quantity $\left|1-\frac{u_{z}^{h}}{\tilde{u}_{z}}\right| \%$ at point $\mathrm{B}$, note the sudden change in convergence when the unphysical coupling is resolved.

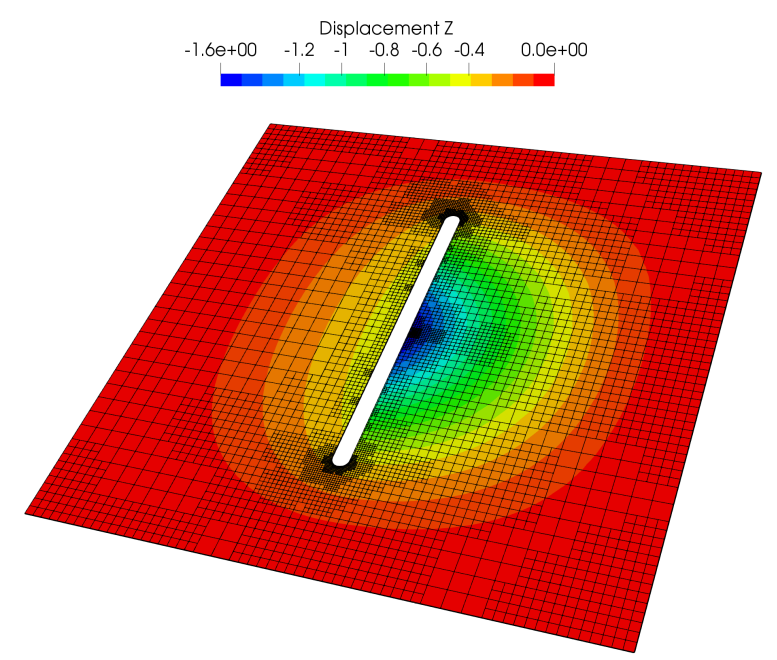

(d) Solution $u_{z}$ obtained at iteration $k=14$, hierarchical B-Splines of degree $p=3$.

Figure 4: Square plate with a thin hole example. 
elements, generally yielding simpler intersections. By doing this, small geometric features induced by the trimming curves can be selectively resolved. For instance, in Figures $5 \mathrm{~d}$ and $5 \mathrm{e}$ the solution and Von Mises stress distribution obtained with $\gamma=p=2$ and $l_{\max }=5$ is shown. This example qualitatively demonstrates the capability of local refinement to reduce the over-constraining effects linked to the weak imposition of Dirichlet-type boundary conditions.

We compare the resultant Von Mises stresses for different meshes in Figure 6. Figure 6b shows the solution obtained with a uniform mesh of 38607 DOFs. The mesh is chosen to have a number of DOFs similar to the locally refined mesh in Figures 5e and 6a, which has 36225 DOFs. As a reference, Figure 6c shows the solution obtained by an overkill mesh with 4026378 DOFs. The locally refined mesh does not have artificially low stress on the constrain curve, similarly to the overkill solution.

\subsection{Localized deformations}

The example given in Figure 1 produces a localized deformation (see Figure 1d) which needs to be properly resolved. We highlight once more that the local refinement strategy employed to remove the unphysical coupling already gives an approximation that is comparable to uniform refinement, but with a considerable reduction in DOFs, where we refer to Figures 2a and 2b, respectively. In Table 1 , the energy and z-displacement (at point $\mathrm{A}$ ) errors are shown with respect to the following reference values $\tilde{E} \approx 0.2211, \tilde{u}_{z} \approx 7.268 \cdot 10^{-3}$. These values were obtained with an overkill solution on a uniformly refined mesh of level $l_{\max }=5$. This numerical experiment confirms that local refinement accurately and efficiently captures both local quantities (such as the solution at point A) and global quantities (the energy of the system) compared to tensor product refinement, where in this particular example $\sim 5$ times less DOFs are required to achieve a comparable level of accuracy.

\begin{tabular}{llll} 
& num. DOFs & energy error $\left(1-\frac{E^{h}}{\tilde{E}}\right) \%$ & z-displ. error at pt. A $\left(1-\frac{u_{z}^{h}}{\tilde{u}_{z}}\right) \%$ \\
\hline tensor product ref. & 193359 & $2.07 \%$ & $1.72 \%$ \\
local ref. & 37215 & $2.88 \%$ & $2.29 \%$
\end{tabular}

Table 1: Comparison of the error in the energy norm and error in the z-displacement at point A against the number of DOFs for tensor product and local refinements. 


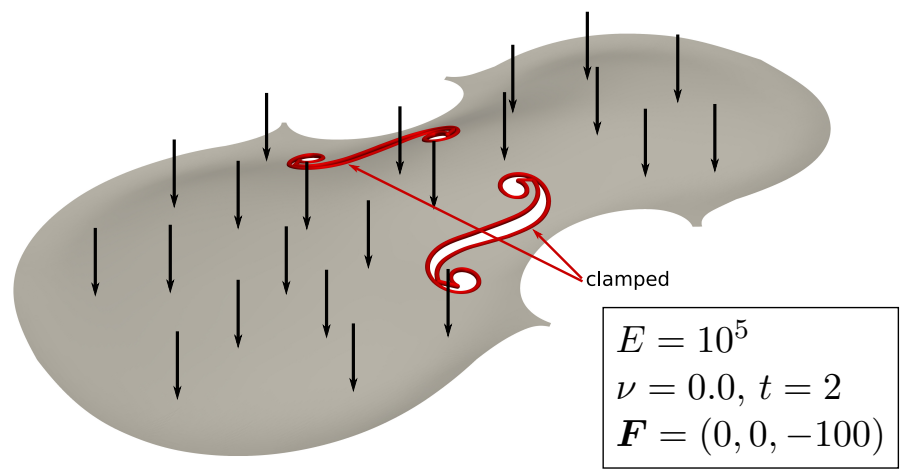

(a) Boundary conditions: clamped (red wire) and distributed load (arrows).

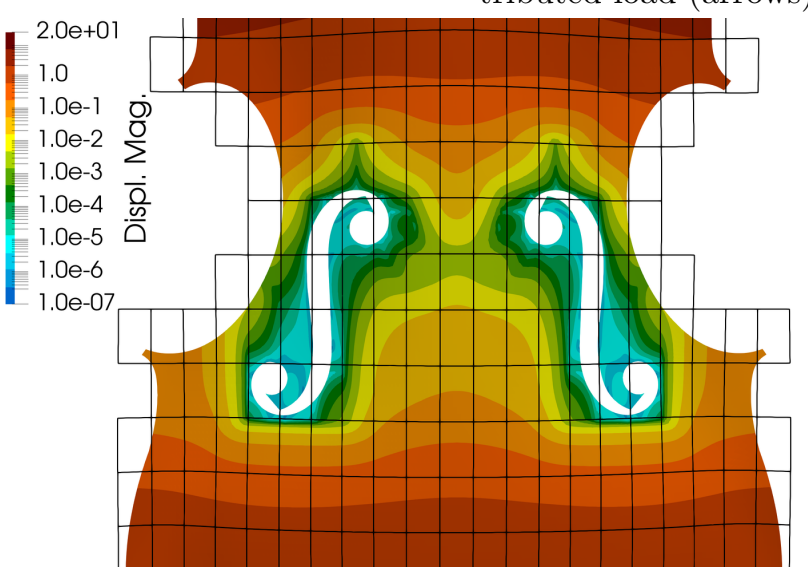

(b) Displacement magnitude (logarithmic scale) on unrefined mesh.

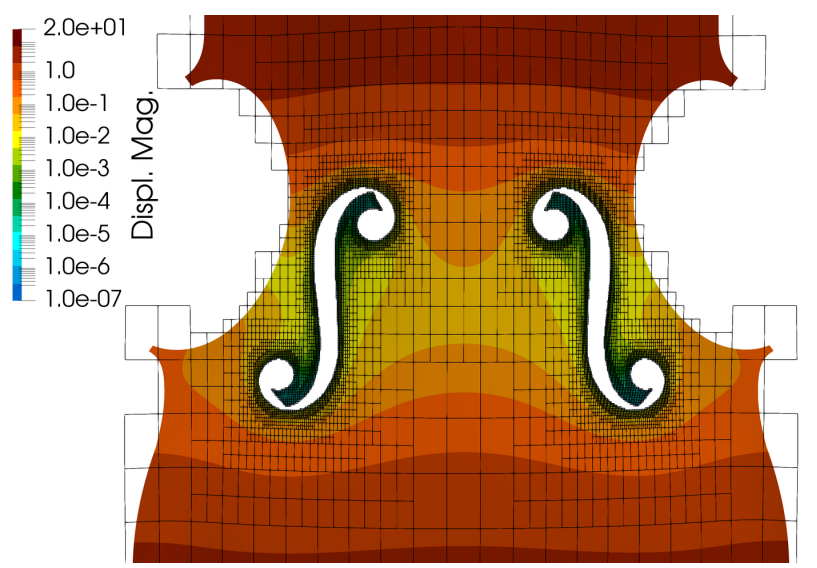

(d) Displacement magnitude (logarithmic scale) on a locally refined mesh.

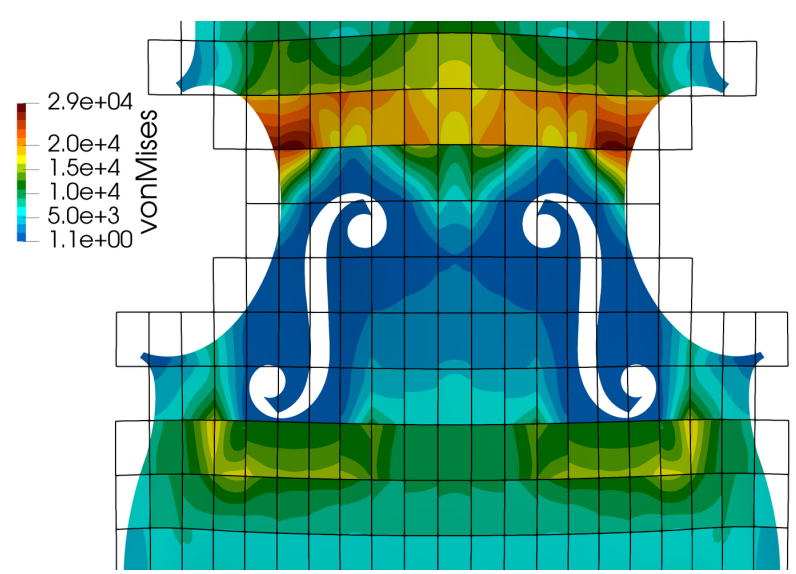

(c) Von Mises stress on unrefined mesh.

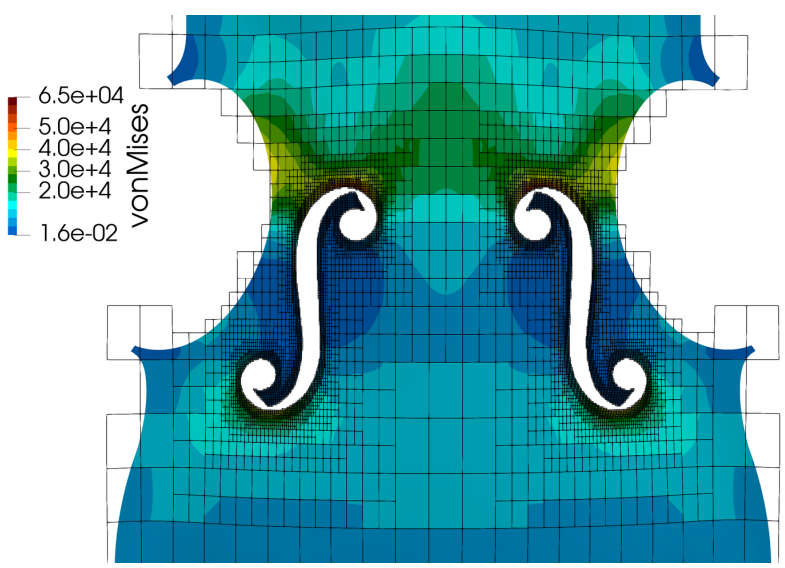

(e) Von Mises stress on a locally refined mesh.

Figure 5: Example of a violin subjected to gravity load. 


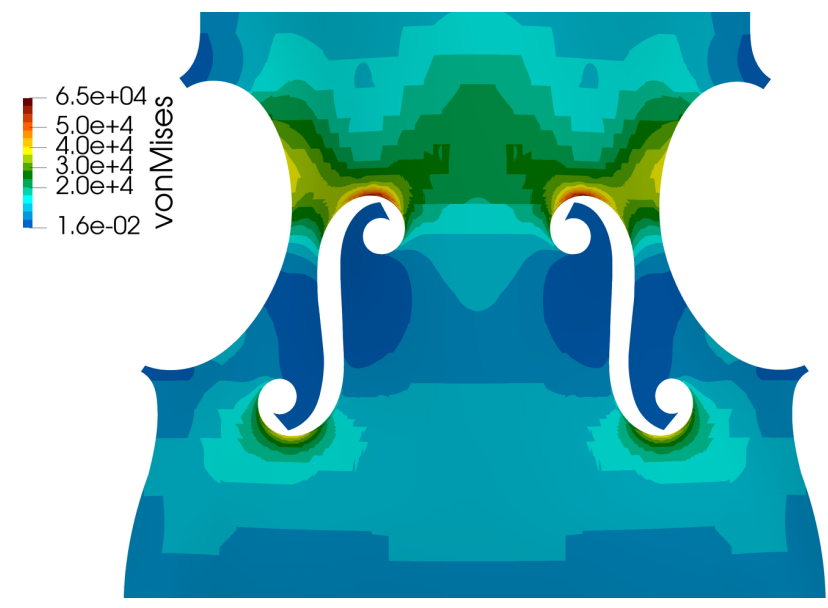

(a) Von Mises on a locally refined mesh as in Figure $5 \mathrm{e}$

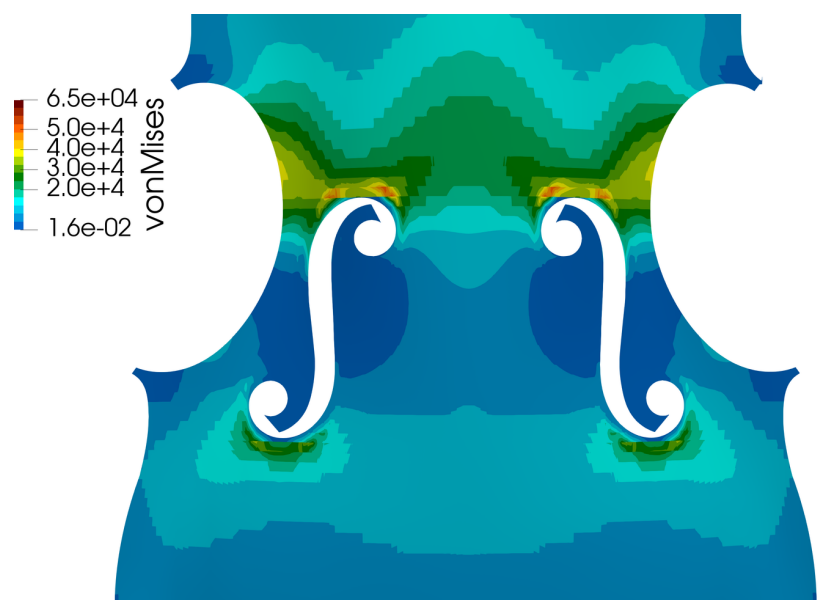

(b) Von Mises on a uniformly refined mesh such that the number of DOFs is similar to Figure $6 \mathrm{a}$.

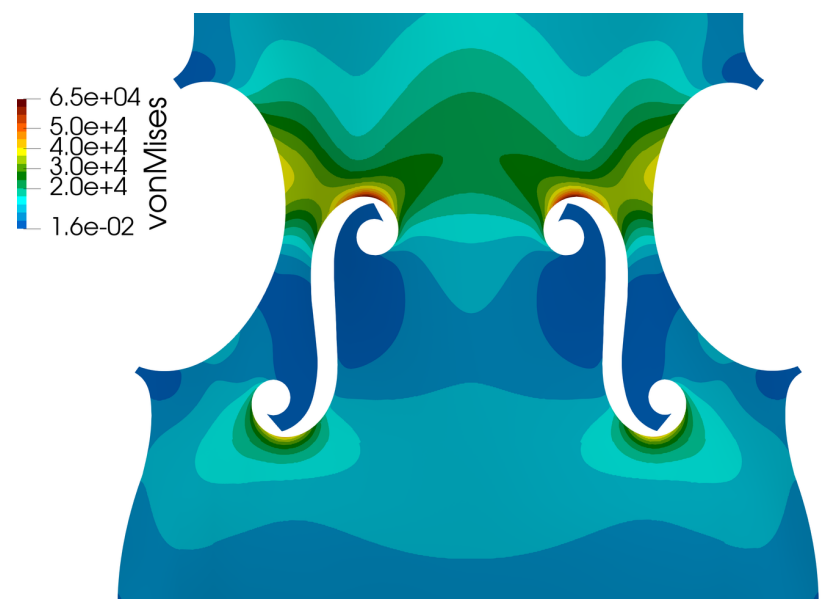

(c) Von Mises on a overkill mesh.

Figure 6: Comparison of Von Mises stresses around the f-holes for different meshes. 


\section{Numerical Examples}

In this section, several examples that show the applicability of the proposed workflow to the locallyrefined analysis of trimmed surfaces are presented. All the geometries used in the following examples have been created in the commercial CAD software Rhinoceros and exported in the STEP file format [33. The parsing of the geometry files and the computations have been performed within the in-house code $\mathrm{AdHoC}++$, exploiting the OpenCascade library for geometric support [49].

\subsection{A first trimmed example with adaptive local refinement}

The first example deals with a geometry created by the intersection of two cylinders. The description of the geometry has been taken from an example presented in [10], where we remark that the two trimmed holes are symmetric with respect to the diagonal of the cylinder, see Figure 7. We apply simply supported boundary conditions to the two big circular ends. Furthermore, the structure is subjected to a unit point load in the middle directed to the negative $\mathrm{z}$-direction, $\boldsymbol{F}=(0,0,-1)^{\top}$. The material parameters are chosen as $E=5 \cdot 10^{7}$ and $\nu=0.0$ for the Young modulus and Poisson ratio, respectively. The shell thickness is set equal to 0.1 . In order to steer an adaptive simulation, the $L^{\infty}$-norm of the numerical gradient has been used as a simple indicator for refining the mesh. In particular, we utilize a method similar to the so-called maximum strategy for marking elements for refinement. Let $\gamma \in(0,1)$ be a user-defined threshold, all elements such that

$$
\left\|\nabla \mathbf{u}_{\mathbf{h}}(\epsilon)\right\|_{L^{\infty}(\epsilon)}>\gamma \max _{\varepsilon}\left\|\nabla \mathbf{u}_{\mathbf{h}}(\varepsilon)\right\|_{L^{\infty}(\varepsilon)}
$$

are marked for refinement, where $\nabla \mathbf{u}_{\mathbf{h}}(\epsilon)$ denotes the numerical gradient restricted to element $\epsilon$. In addition, we always refine elements adjacent to the supports. Despite the simplicity of this error indicator, numerical experiments show that it behaves well in driving an adaptive simulation for the problem at hand, as presented in Figure 8 where we can see that the refinement correctly captures the main characteristics of the solution.

Moreover, for this example we study the convergence of the internal energy and z-displacement under the point load on a series of refined meshes. The reference solution has been obtained from an overkill on a uniformly-refined mesh with 2603682 DOFs, $p=3$, and the results plotted against the number of DOFs are presented in Figure 9. Additionally, let us define the displacement magnitude as:

$$
\left\|\boldsymbol{u}_{h}\right\|_{2}=\sqrt{u_{x}^{2}+u_{y}^{2}+u_{z}^{2}}
$$

The corresponding contour plot is shown in Figure $8 \mathrm{c}$

Finally, we show the integration cells obtained from our reparametrization tool in the proximity of one of the trimming curves, see Figure $8 \mathrm{~b}$. It can be observed that the smart tree integration algorithm performs robustly even when trimmed elements belonging to different levels of the hierarchy are present. We also remark that local refinement simplifies the intersections between the trimming curve and the elements, generally yielding fewer complex cases to be handled.

\subsection{From CAD to analysis of an "engineering" structure}

The next example concerns the simulation of a simplified model, depicted in Figure 10a, of the rooftop of the Rolex Learning Center, the campus library at the École Polytechnique Fédérale de Lausanne (see Figure 10b). Here, we aim at showing the capabilities of the proposed numerical framework for the analysis of complex structures of architectural relevance.

The geometry is modeled as a trimmed NURBS surface of degree $p=3$ composed of $20 \times 20$ knot spans. A planar map of the building has been used to trace all the major structural holes. Then, 150 


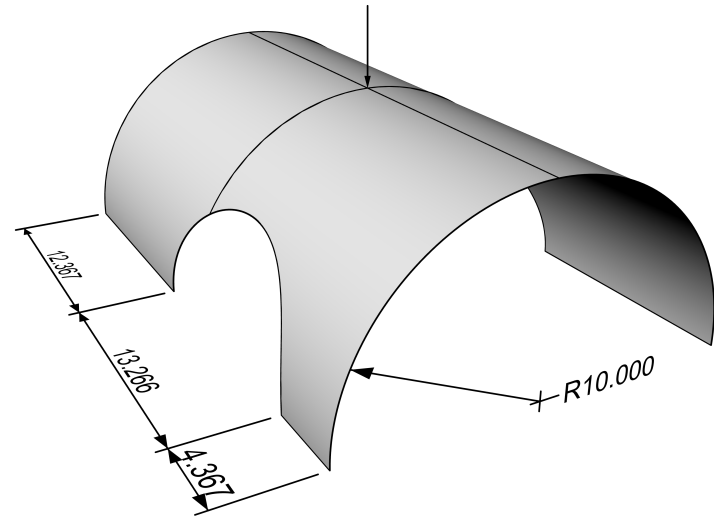

(a) Geometry definition in Rhino.

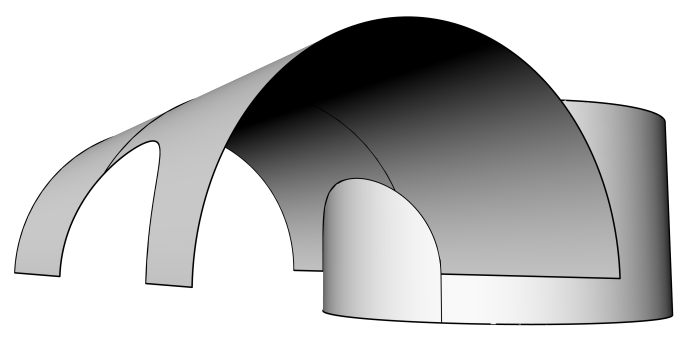

(b) Trimming boolean operation.

Figure 7: Geometry specifications and representation of the trimming operation in Rhino used to create the two trimmed holes, defined as the intersection between two cylinders.

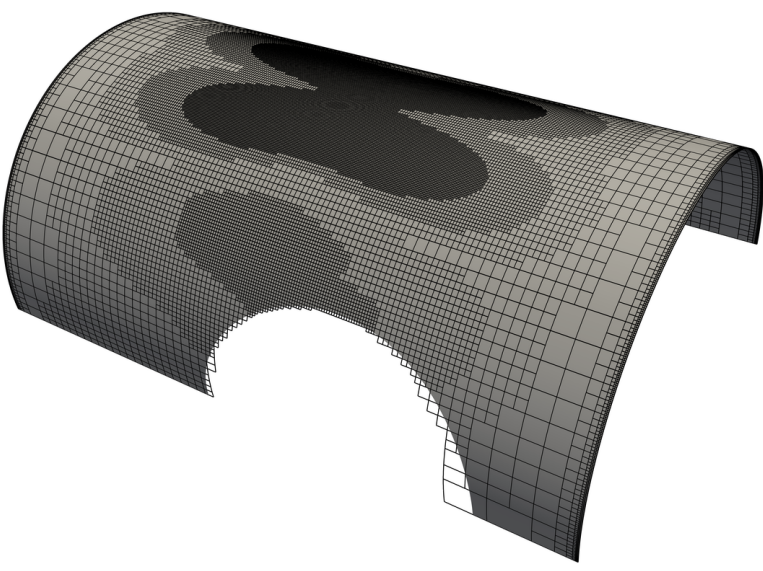

(a) Hierarchical mesh.

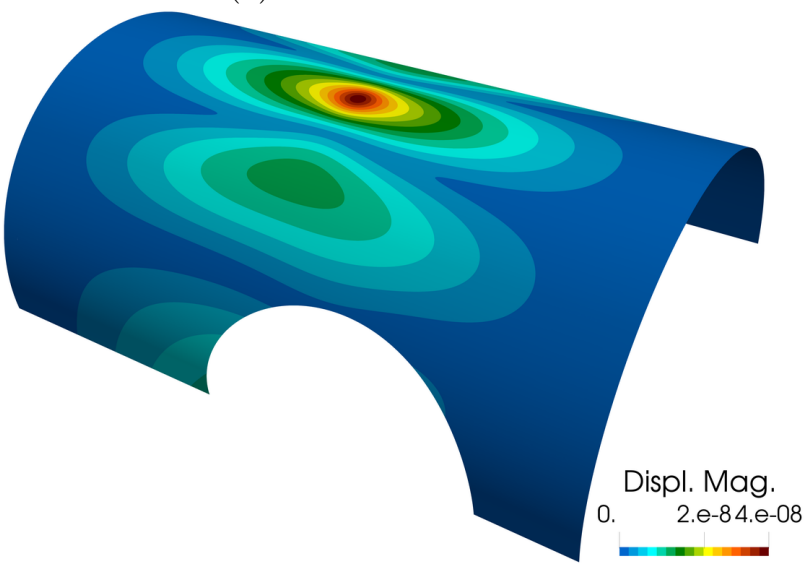

(c) Displacement magnitude.

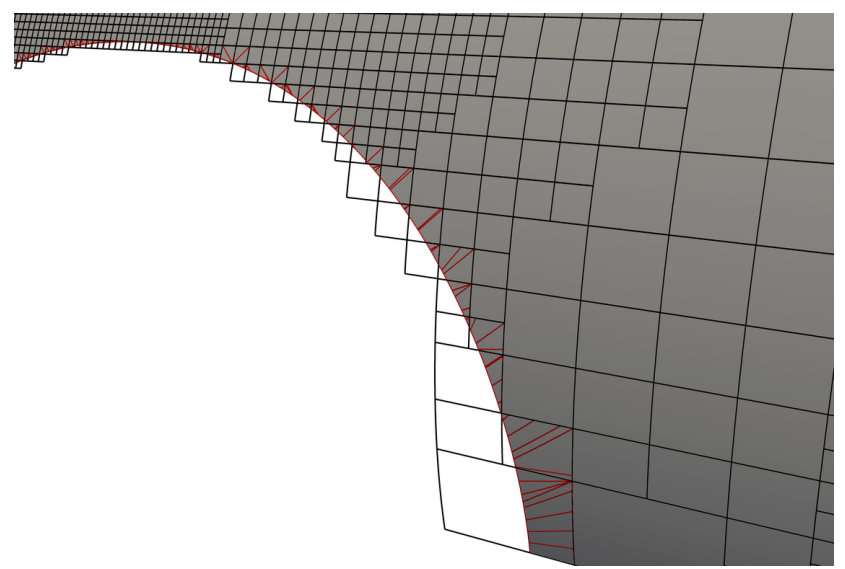

(b) Reparametrized integration cells (red edges).

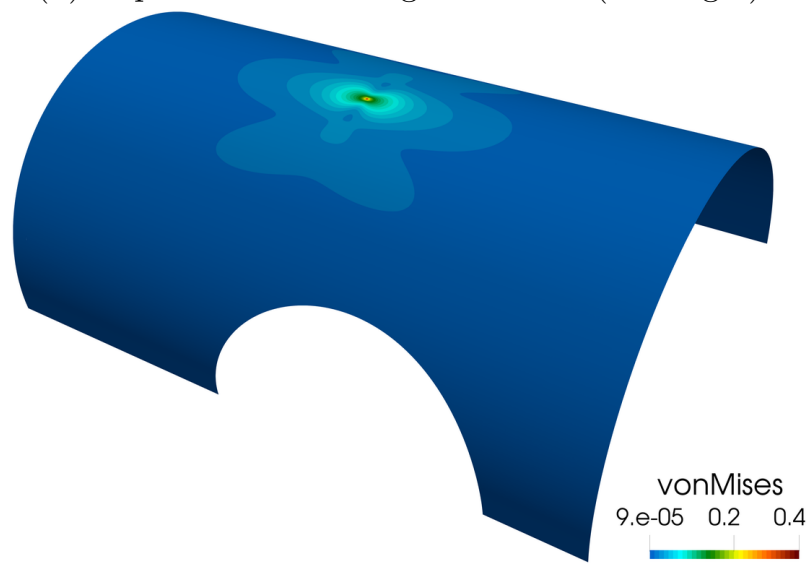

(d) Von Mises stress.

Figure 8: Mesh, displacement magnitude and Von Mises stress obtained after 6 iterations of the adaptive loop, marking parameter $\gamma=0.2$. Solution obtained with hierarchical B-Splines of degree $p=3$. 


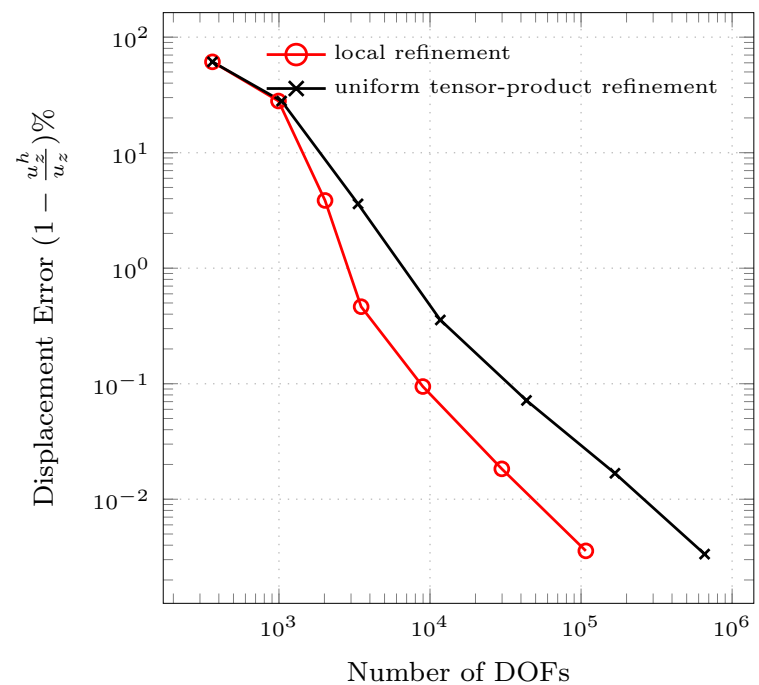

(a) Error in the z-displacement under the point load.

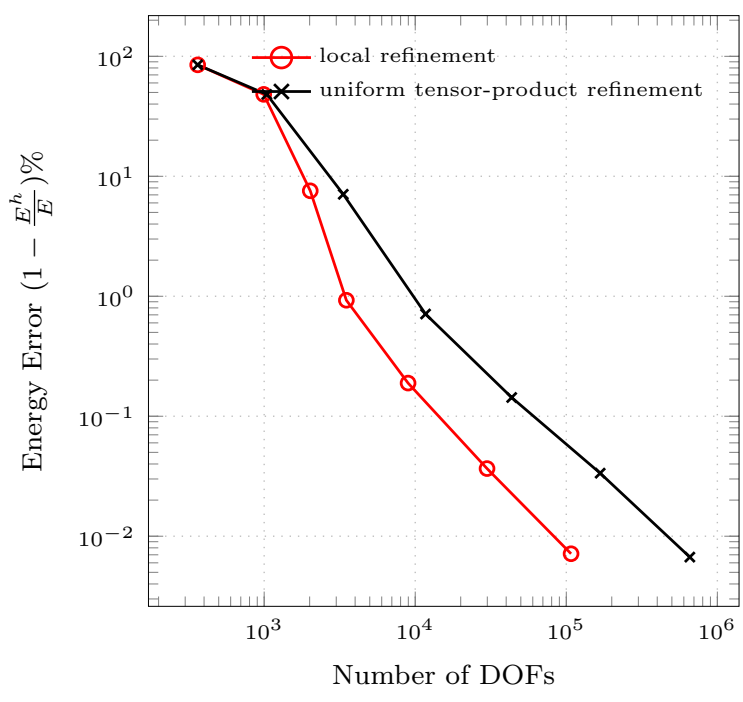

(b) Error measured in the energy norm.

Figure 9: Convergence of the error in displacement and energy obtained with hierarchical B-Splines of degree $p=3$ and marking parameter $\gamma=0.2$.

cylinders representing the pillars have been placed in a $15 \times 10$ Cartesian fashion into the model (those falling outside of the physical domain have been discarded). Their intersection with the surface is used to impose homogeneous Dirichlet boundary conditions on the displacement, therefore modeling the static behavior of the supports. Let us remark the fact that this simplification in the design may not correspond to the actual placement of the pillars. Additionally, we remark that we are aware of the limitation of this model, but in the scope of this work it is used as an illustrative example for the wide range of applicability of the proposed simulation tool, spanning from complex geometrical models to architectural designs. Finally, the material parameters are chosen as $E=40 \cdot 10^{9}, \nu=0.15$ and $t=0.2$ for the Young modulus, Poisson ratio, and thickness of the shell, respectively.

The roof is subjected to its self-weight, set to $\boldsymbol{F}=\left(0,0,-2 \cdot 10^{3}\right)^{\top}$, and in Figure $10 \mathrm{c}$ the displacement magnitude field is depicted, where $k=5$ levels of hierarchical refinement are used to resolve the boundary conditions given by the pillars and the trimming curves. A close-up of the solution in the vicinity of a trimming curve for a coarse uniform tensor-product mesh and a locally refined mesh is depicted in Figure 11. We note a substantial reduction of over-constraining of the solution field close to the pillars in the locally refined case. On one hand, this example clearly motivates local refinement, which is needed to resolve the small scale of the problem (the pillars) while considerably reducing the computational cost. On the other hand, it shows how all the relevant information needed to impose boundary conditions can be taken directly from the CAD model, in the spirit of a full design-through-analysis workflow. 


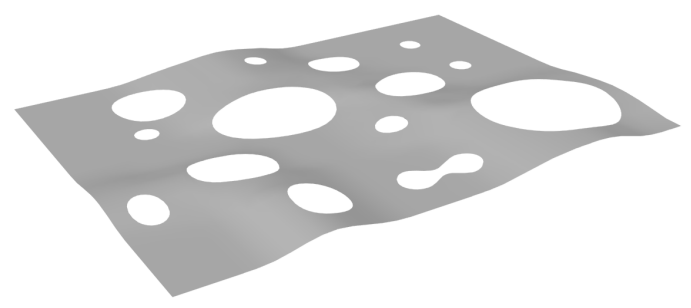

(a) CAD model

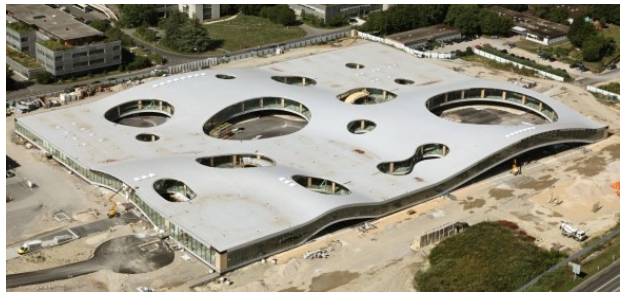

(b) Aerial photo (source: Wikipedia)

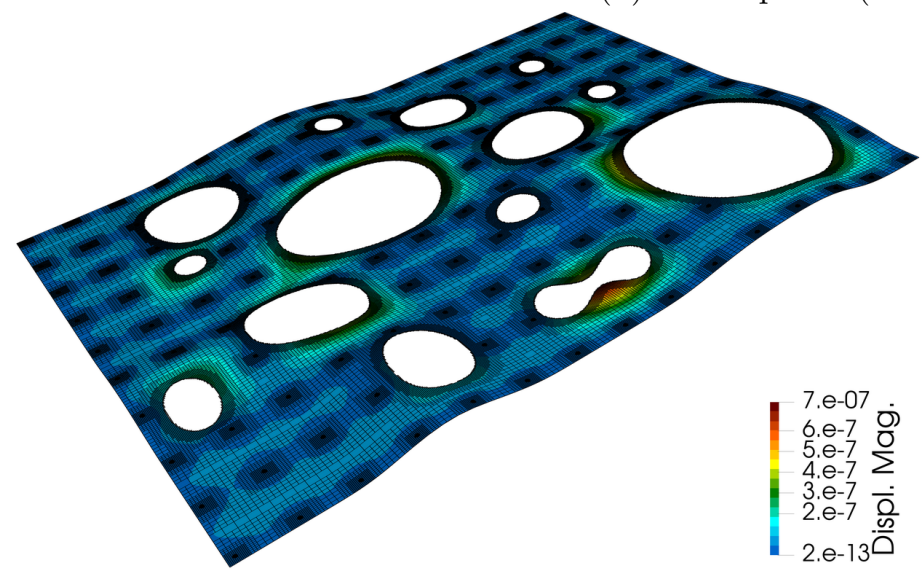

(c) Displacement magnitude.

Figure 10: Geometric model, actual building and solution of the Rolex Learning Center subject to its self-weight. For the solution hierarchical B-Splines of degree $p=3$ and $k=5$ levels of refinement have been used. The geometry contains 150 pillars modeled as the intersection between the untrimmed surface and cylinders directly in Rhinoceros. 


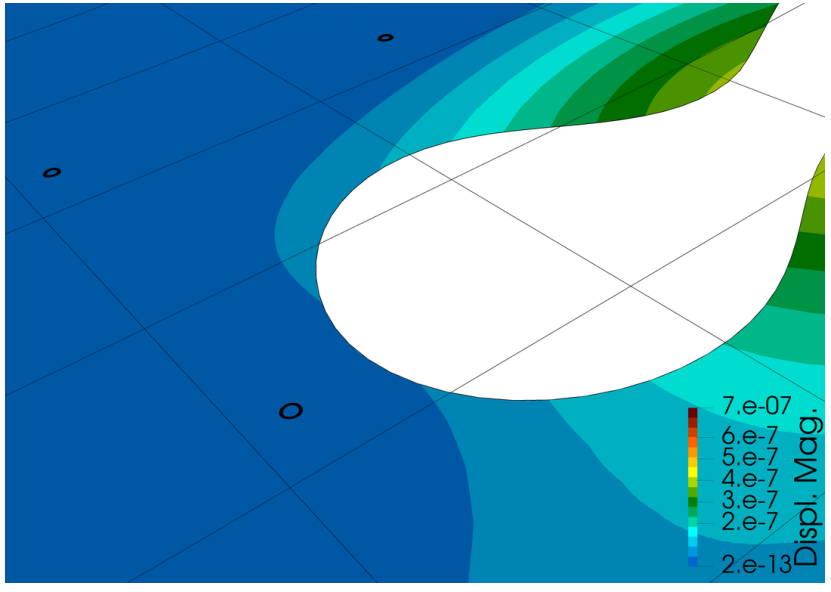

(a) Displacement plot on a coarse mesh.

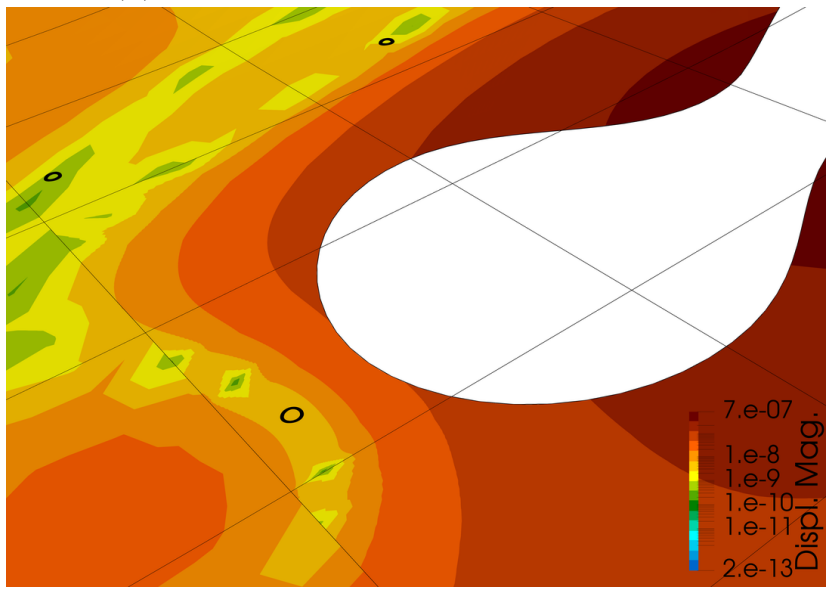

(c) Displacement plot on a coarse mesh (logarithmic scale).

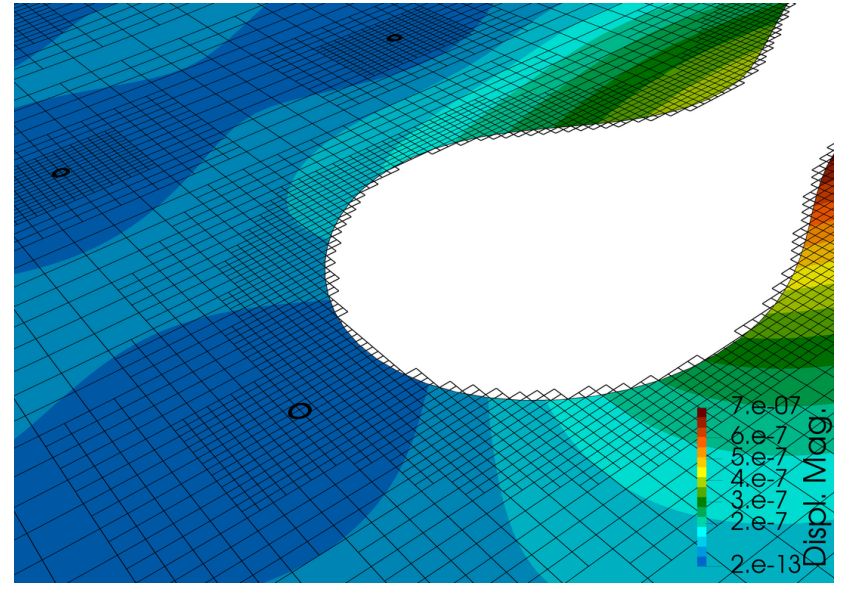

(b) Displacement plot on locally refined mesh.

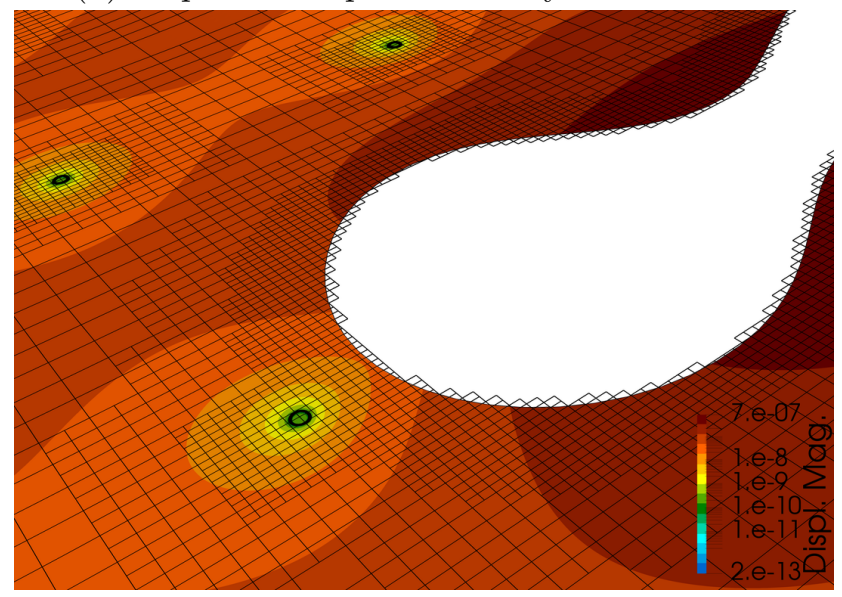

(d) Displacement plot on a locally refined mesh (logarithmic scale).

Figure 11: Zoom on the solution in the proximity of a trimming curve for the Rolex Learning Center example. Note how weakly imposed boundary conditions and geometric features are efficiently resolved by local refinement. 


\section{Conclusions}

In this contribution, we have underlined several of the detrimental effects stemming from trimming of complex B-Rep surfaces in the scope of isogeometric analysis. In particular, we have highlighted the issues related to the weak imposition of Dirichlet-type boundary conditions and to the presence of thin holes in the context of trimmed geometries. Moreover, we have numerically shown how local refinement can efficiently mitigate and eventually eliminate the spurious effects of over-constraining of the solution space in the proximity of weakly constrained trimming curves. Furthermore, we have presented a simple algorithmic way to remove the unphysical coupling of basis functions across "small" geometrical features. Finally, we have tested the proposed analysis pipeline on surfaces with complex features and details defined at different scales, demonstrating the flexibility and vast range of applicability of the combined methods.

To conclude, the proposed workflow aims at reducing the gap between design and analysis by efficiently bringing together several methodologies to tackle problems defined on complex trimmed geometries, for which local refinement is a necessary ingredient. This was achieved by exploiting the properties of hierarchical B-Splines. We have also shed some light on the detrimental effects linked to thin geometrical features and to over-constraining of elements due to weak imposition of boundary conditions, where again local refinement has shown its potential as a simple and effective remedy.

\section{Acknowledgements}

The author L. Coradello gratefully acknowledges the support of the European Research Council, via the ERC AdG project CHANGE (no. 694515). A. Reali has been partially supported by the MIURPRIN project XFAST-SIMS (no. 20173C478N). The authors D. D'Angella and A. Reali acknowledge the support of the TUM Institute for Advanced Study, funded by the German Excellence Initiative and the European Union Seventh Framework Programme under grant agreement number 291763. D. D'Angella acknowledges the support of the Deutsche Forschungsgemeinschaft in the Priority Program 1748 "Reliable simulation techniques in solid mechanics. Development of non-standard discretization methods, mechanical and mathematical analysis" under the project RA624/27-2.

\section{References}

[1] Special Issue on Isogeometric Analysis: Progress and Challenges. Computer Methods in Applied Mechanics and Engineering. Elsevier, 2017.

[2] A. Abedian, J. Parvizian, A. Düster, and E. Rank. Finite cell method compared to h-version finite element method for elasto-plastic problems. Applied Mathematics and Mechanics, 35(10): 1239-1248, 2014-10-01. 00002.

[3] P. Antolin, A. Buffa, and M. Martinelli. Isogeometric Analysis on V-reps: First results. Computer Methods in Applied Mechanics and Engineering, 355:976 - 1002, 2019.

[4] C. Apprich, K. Höllig, J. Hörner, A. Keller, and E. Nava Yazdani. Finite Element Approximation with Hierarchical B-Splines. In J.-D. Boissonnat, A. Cohen, O. Gibaru, C. Gout, T. Lyche, M.-L. Mazure, and L. L. Schumaker, editors, Curves and Surfaces, pages 1-15, Cham, 2015. Springer International Publishing.

[5] I. Babuška. The finite element method with penalty. Mathematics of Computation, 27(122): 221-228, 1973. 
[6] Y. Bazilevs, V. Calo, J. Cottrell, J. Evans, T. J. R. Hughes, S. Lipton, M. Scott, and T. Sederberg. Isogeometric analysis using T-splines. Computer Methods in Applied Mechanics and Engineering, 199(5):229 - 263, 2010. Computational Geometry and Analysis.

[7] T. Bog, N. Zander, S. Kollmannsberger, and E. Rank. Normal contact with high order finite elements and a fictitious contact material. Computers \& Mathematics with Applications, 70(7): 1370-1390, 2015-10.

[8] M. Breitenberger, A. Apostolatos, B. Philipp, R. Wüchner, and K.-U. Bletzinger. Analysis in computer aided design: Nonlinear isogeometric B-Rep analysis of shell structures. Computer Methods in Applied Mechanics and Engineering, 284:401 - 457, 2015. Isogeometric Analysis Special Issue.

[9] M. Carraturo, C. Giannelli, A. Reali, and R. Vázquez. Suitably graded THB-spline refinement and coarsening: Towards an adaptive isogeometric analysis of additive manufacturing processes. Computer Methods in Applied Mechanics and Engineering, 348:660 - 679, 2019.

[10] H. Casquero, L. Liu, Y. Zhang, A. Reali, J. Kiendl, and H. Gómez. Arbitrary-degree T-splines for isogeometric analysis of fully nonlinear Kirchhoff-Love shells. Computer-Aided Design, 82: 140 - 153, 2017. Isogeometric Design and Analysis.

[11] P. Ciarlet. The Finite Element Method for Elliptic Problems. Society for Industrial and Applied Mathematics, 2002.

[12] F. Cirak. Subdivision shells. In C. A. Motasoares, J. A. C. Martins, H. C. Rodrigues, J. A. C. Ambrósio, C. A. B. Pina, C. M. Motasoares, E. B. R. Pereira, and J. Folgado, editors, III European Conference on Computational Mechanics, pages 395-395, Dordrecht, 2006. Springer Netherlands.

[13] L. Coradello, P. Antolin, R. Vázquez, and A. Buffa. Adaptive isogeometric analysis on twodimensional trimmed domains based on a hierarchical approach, 2019.

[14] J. A. Cottrell, T. J. R. Hughes, and Y. Bazilevs. Isogeometric Analysis. John Wiley \& Sons, Ltd, Chichester, UK, August 2009.

[15] L. B. da Veiga, A. Buffa, G. Sangalli, and R. Vázquez. Mathematical analysis of variational isogeometric methods. Acta Numerica, 23:157-287, 2014.

[16] D. D'Angella, S. Kollmannsberger, E. Rank, and A. Reali. Multi-level Bézier extraction for hierarchical local refinement of isogeometric analysis. Computer Methods in Applied Mechanics and Engineering, Jan 2018.

[17] F. de Prenter, C. Lehrenfeld, and A. Massing. A note on the stability parameter in Nitsche's method for unfitted boundary value problems. Computers $\& 3$ Mathematics with Applications, 75 (12):4322 - 4336, 2018.

[18] T. Dokken, T. Lyche, and K. F. Pettersen. Polynomial splines over locally refined box-partitions. Computer Aided Geometric Design, 30(3):331 - 356, 2013.

[19] A. Düster, J. Parvizian, Z. Yang, and E. Rank. The finite cell method for three-dimensional problems of solid mechanics. Computer Methods in Applied Mechanics and Engineering, 197 (45-48):3768-3782, 2008-08-15.

[20] M. Elhaddad, N. Zander, S. Kollmannsberger, A. Shadavakhsh, V. Nübel, and E. Rank. Finite Cell Method: High-Order Structural Dynamics for Complex Geometries. International Journal of Structural Stability and Dynamics, page 1540018, 2015-04-14. 00000. 
[21] D. R. Forsey and R. H. Bartels. Hierarchical B-spline Refinement. In Proceedings of the 15th Annual Conference on Computer Graphics and Interactive Techniques, SIGGRAPH '88, pages 205-212, New York, NY, USA, 1988. ACM.

[22] E. M. Garau and R. Vázquez. Algorithms for the implementation of adaptive isogeometric methods using hierarchical B-splines. Applied Numerical Mathematics, 123:58 - 87, 2018.

[23] C. Giannelli, B. Jüttler, and H. Speleers. THB-splines: The truncated basis for hierarchical splines. Computer Aided Geometric Design, 29(7):485-498, October 2012.

[24] C. Giannelli, B. Jüttler, S. K. Kleiss, A. Mantzaflaris, B. Simeon, and J. Špeh. THB-splines: An effective mathematical technology for adaptive refinement in geometric design and isogeometric analysis. Computer Methods in Applied Mechanics and Engineering, 299:337-365, February 2016.

[25] H. Gómez, V. M. Calo, Y. Bazilevs, and T. J. R. Hughes. Isogeometric analysis of the CahnHilliard phase-field model. Computer Methods in Applied Mechanics and Engineering, 197(49): $4333-4352,2008$.

[26] G. Greiner and K. Hormann. Interpolating and Approximating Scattered 3D-data with Hierarchical Tensor Product B-Splines. In In Surface Fitting and Multiresolution Methods, pages 163-172. Vanderbilt University Press, 1997.

[27] Y. Guo and M. Ruess. Weak Dirichlet boundary conditions for trimmed thin isogeometric shells. Computers \& Mathematics with Applications, 70(7):1425 - 1440, 2015. High-Order Finite Element and Isogeometric Methods.

[28] Y. Guo, J. Heller, T. J. R. Hughes, M. Ruess, and D. Schillinger. Variationally consistent isogeometric analysis of trimmed thin shells at finite deformations, based on the step exchange format. Computer Methods in Applied Mechanics and Engineering, 336:39 - 79, 2018.

[29] A. J. Herrema, E. L. Johnson, D. Proserpio, M. C. Wu, J. Kiendl, and M.-C. Hsu. Penalty coupling of non-matching isogeometric Kirchhoff-Love shell patches with application to composite wind turbine blades. Computer Methods in Applied Mechanics and Engineering, 346:810 - 840, 2019 .

[30] K. Höllig. Finite element methods with B-splines, volume 26 of Frontiers in Applied Mathematics. Society for Industrial and Applied Mathematics (SIAM), Philadelphia, PA, 2003.

[31] K. Höllig, J. Hörner, and A. Hoffacker. Finite Element Analysis with B-Splines: Weighted and Isogeometric Methods. In J.-D. Boissonnat, P. Chenin, A. Cohen, C. Gout, T. Lyche, M.-L. Mazure, and L. Schumaker, editors, Curves and Surfaces, pages 330-350, Berlin, Heidelberg, 2012. Springer Berlin Heidelberg.

[32] T. J. R. Hughes, J. A. Cottrell, and Y. Bazilevs. Isogeometric analysis: CAD, finite elements, NURBS, exact geometry and mesh refinement. Computer Methods in Applied Mechanics and Engineering, 194(39-41):4135-4195, October 2005.

[33] ISO 10303-11:1994. Industrial automation systems and integration - Product data representation and exchange. Standard, International Organization for Standardization, Geneva, CH, March 1994.

[34] J. Kiendl, K.-U. Bletzinger, J. Linhard, and R. Wüchner. Isogeometric shell analysis with Kirchhoff-Love elements. Computer Methods in Applied Mechanics and Engineering, 198(49): $3902-3914,2009$. 
[35] J. Kiendl, M.-C. Hsu, M. C. Wu, and A. Reali. Isogeometric Kirchhoff-Love shell formulations for general hyperelastic materials. Computer Methods in Applied Mechanics and Engineering, $291: 280-303,2015$.

[36] J. Kiendl, M. Ambati, L. D. Lorenzis, H. Gomez, and A. Reali. Phase-field description of brittle fracture in plates and shells. Computer Methods in Applied Mechanics and Engineering, 312: 374 - 394, 2016. Phase Field Approaches to Fracture.

[37] R. Kraft. Adaptive and Linearly Independent Multilevel B-splines. In Surface Fitting and Multiresolution Methods. Vanderbilt University Press, 1997.

[38] L. Kudela, N. Zander, T. Bog, S. Kollmannsberger, and E. Rank. Efficient and accurate numerical quadrature for immersed boundary methods. Advanced Modeling and Simulation in Engineering Sciences, 2(1):10, 2015.

[39] L. Kudela, N. Zander, S. Kollmannsberger, and E. Rank. Smart octrees: accurately integrating discontinuous functions in 3d. Computer Methods in Applied Mechanics and Engineering, Apr 2016 .

[40] L. Leonetti, D. Magisano, A. Madeo, G. Garcea, J. Kiendl, and A. Reali. A simplified KirchhoffLove large deformation model for elastic shells and its effective isogeometric formulation. Computer Methods in Applied Mechanics and Engineering, 354:369 - 396, 2019.

[41] L. Liu, Y. Zhang, T. J. R. Hughes, M. A. Scott, and T. W. Sederberg. Volumetric t-spline construction using boolean operations. Engineering with Computers, 30(4):425-439, Oct 2014.

[42] L. Liu, Y. J. Zhang, and X. Wei. Weighted T-splines with application in reparameterizing trimmed nurbs surfaces. Computer Methods in Applied Mechanics and Engineering, 295:108 $126,2015$.

[43] G. Lorenzo, M. Scott, K. Tew, T. J. R. Hughes, and H. Gómez. Hierarchically refined and coarsened splines for moving interface problems, with particular application to phase-field models of prostate tumor growth. Computer Methods in Applied Mechanics and Engineering, 319:515 $-548,2017$.

[44] B. Marussig and T. J. R. Hughes. A review of trimming in isogeometric analysis: Challenges, data exchange and simulation aspects. Archives of Computational Methods in Engineering, 25 (4):1059-1127, Nov 2018.

[45] J. Parvizian, A. Düster, and E. Rank. Finite cell method. Computational Mechanics, 41(1): 121-133, 2007-04.

[46] E. Rank, S. Kollmannsberger, C. Sorger, and A. Düster. Shell Finite Cell Method: A high order fictitious domain approach for thin-walled structures. Computer Methods in Applied Mechanics and Engineering, 200(45-46):3200-3209, 2011-10.

[47] A. Reali and H. Gómez. An isogeometric collocation approach for Bernoulli-Euler beams and Kirchhoff plates. Computer Methods in Applied Mechanics and Engineering, 284:623 - 636, 2015. Isogeometric Analysis Special Issue.

[48] M. Ruess, D. Tal, N. Trabelsi, Z. Yosibash, and E. Rank. The finite cell method for bone simulations: verification and validation. Biomechanics and modeling in mechanobiology, 11 (3-4):425-37, 2012-03.

[49] J. Russell and R. Cohn. Open Cascade Technology. 2012. 
[50] D. Schillinger, L. Dedé, M. A. Scott, J. A. Evans, M. J. Borden, E. Rank, and T. J. R. Hughes. An isogeometric design-through-analysis methodology based on adaptive hierarchical refinement of NURBS, immersed boundary methods, and T-spline CAD surfaces. Computer Methods in Applied Mechanics and Engineering, 249-252:116 - 150, 2012. Higher Order Finite Element and Isogeometric Methods.

[51] D. Schillinger, M. Ruess, N. Zander, Y. Bazilevs, A. Düster, and E. Rank. Small and large deformation analysis with the p- and B-spline versions of the Finite Cell Method. Computational Mechanics, 50(4):445-478, 2012-02.

[52] R. Schmidt, J. Kiendl, K.-U. Bletzinger, and R. Wüchner. Realization of an integrated structural design process: analysis-suitable geometric modelling and isogeometric analysis. Computing and Visualization in Science, 13(7):315-330, Oct 2010.

[53] R. Schmidt, R. Wüchner, and K.-U. Bletzinger. Isogeometric analysis of trimmed NURBS geometries. Computer Methods in Applied Mechanics and Engineering, 241-244:93 - 111, 2012.

[54] M. Utku and G. Carey. Boundary penalty techniques. Computer Methods in Applied Mechanics and Engineering, 30(1):103 - 118, 1982.

[55] A. V. Vuong, C. Giannelli, B. Jüttler, and B. Simeon. A hierarchical approach to adaptive local refinement in isogeometric analysis. Computer Methods in Applied Mechanics and Engineering, 200(49-52):3554-3567, December 2011.

[56] B. Wassermann, S. Kollmannsberger, T. Bog, and E. Rank. From geometric design to numerical analysis: A direct approach using the Finite Cell Method on Constructive Solid Geometry. Computers $\&$ Mathematics with Applications, 74(7):1703 - 1726, 2017. High-Order Finite Element and Isogeometric Methods 2016.

[57] N. Zander, S. Kollmannsberger, M. Ruess, Z. Yosibash, and E. Rank. The Finite Cell Method for linear thermoelasticity. Computers \& Mathematics with Applications, 64(11):3527-3541, 2012-12. 